\title{
Die Auswirkungen der DSGVO auf das zivilrechtliche Äußerungsrecht
}

JProf. Dr. Anne Lauber-Rönsberg, LL.M.*

\author{
I. Einleitung \\ 399 \\ II. Unterschiede zwischen Daten- \\ schutz- und Äußerungsrecht ... 400 \\ III. Rechtslage vor der DSGVO ..... 403 \\ 1. Art. 9 Datenschutz- \\ RL 95/46 \\ 2. Deutsche Rechtslage \\ a) Medienprivileg......... 405 \\ aa) Tatbestandsvoraus- \\ setzungen .......... 406 \\ bb) Äußerungsrecht als \\ „Lückenfüller“....... 408 \\ b) Subsidiarität des BDSG \\ gegenüber dem Äuße- \\ rungsrecht

IV. Rechtslage seit Anwendbarkeit der DSGVO

1. Einschränkung des Anwendungsbereichs bei Datenverarbeitungen für persönliche oder familiäre Tätigkeiten ... 411

2. Kein postmortaler Schutz durch die DSGVO

3. Öffnungsklausel(n) des Art. 85 DSGVO

a) Datenverarbeitungen zu journalistischen, wissenschaftlichen, künstlerischen und literarischen Zwecken, Art. 85 Abs. 2 DSGVO.... 413

aa) Tatbestandsvoraussetzungen

(1) Journalistische Zwecke.... 414
(2) Künstlerische und literarische

Zwecke......... 415

(3) Wissenschaftliche Zwecke

bb) Umfang der Öffnungsklausel: Sanktionen?

b) Datenverarbeitungen (auch) zu anderen Zwecken, Art. 85 Abs. 1

DSGVO

aa) Art. 85 Abs. 1 DSGVO als bloßer Anpassungsauftrag?

bb) Einschränkung des mitgliedstaatlichen Gestaltungsspielraums im Rahmen anderer Öffnungsklauseln?

cc) Art. 85 Abs. 1 DSGVO als eigenständige Öffnungsklausel...

V. Auswirkungen auf das Äußerungsrecht

1. Datenverarbeitungen zu journalistischen Zwecken . 422

a) Regelungen im Landespresserecht. 422

b) Regelungen im Äußerungsrecht 424

aa) Recht am eigenen Bild 425

* Die Verf. ist Juniorprofessorin für Bürgerliches Recht, Medien- und Datenschutzrecht an der Juristischen Fakultät der TU Dresden. Der Beitrag ist die ausgearbeitete Fassung eines Vortrags, den die Verfasserin am 29.6.2018 am Institut für Urheber- und Medienrecht in München hielt. 
bb) Allgemeines Persön-

lichkeitsrecht. 427

2. Datenverarbeitungen zu künstlerischen Zwecken 428

a) Regelungen in den Landesdatenschutzgesetzen

b) Regelungen im Äußerungsrecht

3. Datenverarbeitungen zu nicht in Art. 85 Abs. 2 DSGVO aufgeführten Zwecken a) Szenario 1: Art. 85 Abs. 1 DSGVO als Öffnungsklausel 430

b) Szenario 2: Art. 85 Abs. 1 DSGVO keine Öffnungsklausel

aa) Zulässigkeit von Datenverarbeitungen ....

bb) Folgefragen

VI. Fazit .......................... 434

\begin{abstract}
Bislang wurde der rechtliche Rahmen für die Verarbeitung personenbezogener Daten zu journalistischen oder künstlerischen Zwecken, im Rahmen von Öffentlichkeitsarbeit und Werbung sowie bei Kommunikationsbeiträgen von Privatpersonen z.B. in sozialen Netzwerken in der Regel durch das Äußerungsrecht - insbesondere das allgemeine Persönlichkeitsrecht gemäß $\$ 823$ Abs. 1 BGB i.V.m. Art. 2 Abs. 1, 1 Abs. 1 GG und spezielle Persönlichkeitsrechte wie das Recht am eigenen Bild gemäß $\S \S 22 \mathrm{ff}$. KUG - vorgegeben. Mit der Anwendbarkeit der DSGVO zum 25.5.2018 ist eine Diskussion darüber entbrannt, ob derartige Kommunikationsvorgänge mittlerweile an den datenschutzrechtlichen Vorgaben zu messen sind und inwieweit dem nationalen Gesetzgeber Reformoptionen zur Anpassung des nationalen Rechts zur Verfügung stehen. Der Beitrag stellt zunächst die Vorgaben der DSGVO, insbesondere im Rahmen von Art. 85 Abs. 1 und Abs. 2 DSGVO, dar und geht auf die Anpassungen der landesrechtlichen Regelungen ein. Sodann werden die Auswirkungen der neuen Rechtslage auf die äußerungsrechtlichen Rahmenbedingungen für Datenverarbeitungen zu journalistischen Zwecken, zu künstlerischen Zwecken und im Zusammenhang mit sonstigen Meinungsäußerungen, z.B. im Rahmen der Öffentlichkeitsarbeit oder bei privat motivierten Kommunikationsvorgängen, diskutiert.
\end{abstract}

\title{
I. Einleitung
}

Den Hintergrund der aktuellen Diskussionen bildet das Spannungsverhältnis zwischen dem Persönlichkeitsschutz (Art. 2 Abs. 1 i.V.m. Art. 1 Abs. 1 GG, Art. 8 EMRK, Art. 7,8 GRCh) einerseits und den Kommunikationsfreiheiten sowie der Kunstfreiheit (Art. 5 Abs. 1, Abs. 3 GG, Art. 10 EMRK, Art. 11, 13 GRCh) andererseits. Während der Persönlichkeitsschutz dem Einzelnen ermöglichen soll, selbst über Preisgabe und Verwendung seiner personenbezogenen Daten durch Dritte zu entscheiden, zielen die Kommunikationsfreiheiten auf Aufklärung und Freiheit von Kommunikationsvorgängen, die Kunstfreiheit auf Gewährleistung der Freiheit des Schaffensprozesses als auch der öffentlichen Darbietung 
und Verbreitung des Kunstwerks. Zwischen diesen für eine demokratische Gesellschaft konstitutiven, gleichrangigen Grundrechtspositionen ist ein angemessener Ausgleich i.S. einer praktischen Konkordanz zu schaffen.

Fraglich ist aber, ob dieser Ausgleich nach Inkrafttreten der DSGVO auf einfachgesetzlicher Ebene im Rahmen des Äußerungsrechts oder des Datenschutzrechts zu gewährleisten ist. Sowohl das Äußerungs- als auch das Datenschutzrecht sind dem Persönlichkeitsschutz i.w.S. zuzuordnen; z.T. wird auch der Begriff des Rechts auf informationelle Selbstbestimmung als Oberbegriff verwendet. ${ }^{1}$

Grundsätzlich ist der Anwendungsbereich beider Regelungsregime eröffnet, wenn Personenmerkmale verwendet werden, die zumindest ein Teil der Adressaten auf eine identifizierbare Person zurückführen kann. Die Anwendbarkeit der DSGVO bzw. der nationalen Datenschutzgesetze setzt die Verarbeitung personenbezogener Daten voraus (Art. 2 Abs. 1 DSGVO). ${ }^{2}$ Personenbezogene Daten sind alle Informationen, die sich auf eine identifizierte oder identifizierbare natürliche Person beziehen (Art. 4 Nr. 1 DSGVO). Hierunter fallen auch Fotografien und Filmaufnahmen, die die Identifikation der betroffenen Person ermöglichen. ${ }^{3}$ Zugleich liegt ein Eingriff in spezielle Persönlichkeitsrechte wie das Recht am eigenen Bild gemäß $§ 22 \mathrm{KUG}^{4}$ bzw. das allgemeine Persönlichkeitsrecht ${ }^{5}$ vor.

\section{Unterschiede zwischen Datenschutz- und Äußerungsrecht}

Der Frage, ob ein Ausgleich zwischen den widerstreitenden Rechtspositionen innerhalb des Datenschutz- oder des Äußerungsrechts gewährleistet wird, kommt eine große praktische Bedeutung zu, da zwischen den beiden Rechtsregimen erhebliche Unterschiede bestehen.

Die Entstehung des privatrechtlichen Äußerungsrechts wurde wesentlich durch die Gefährdungen für den Persönlichkeitsschutz durch private Akteure, die mit der Entwicklung moderner Medientechnologien wie der Fotografie einhergingen, motiviert. ${ }^{6}$ Das durch die Rechtsprechung geprägte allgemeine Persönlichkeitsrecht wird durch $\S 823$ Abs. 1 BGB

1 Das solchermaßen verstandene Recht auf informationelle Selbstbestimmung schließt sowohl den informatorischen Schutz der Privatsphäre mit dem Ziel einer informationellen Abschottung als auch das Recht am eigenen Wort und das Recht am eigenen Bild ein, s. Britz, Freie Entfaltung durch Selbstdarstellung, 2007, S. 71 f.; s. auch Di Fabio, in: Maunz/Dürig/u.a., GG, 83. EL April 2018, Art. 2 Abs. 1 Rn. 193: „Recht am eigenen Bild (...) als Konkretisierung des Rechts auf informationelle Selbstbestimmung“.

$2 \mathrm{Zu}$ dem Begriff der Verarbeitung Klein, Personenbilder im Spannungsfeld von Datenschutzgrundverordnung und Kunsturhebergesetz, 2017, S. 29 ff.; zu Art. 2 Datenschutz-RL 95/46 EuGH, Urt. v. 10.7.2018, C-25/17, ZD 2018, 469 Rn. 52 ff. - Zeugen Jehovas.

3 EuGH, Urt. v. 11.12.2014, C-212/13, NJW 2015, 463 Rn. 22 - Ryneš zu Art. 2 lit. a RL 95/46.

4 S. statt aller BGH, Urt. v. 26.6.1979, VI ZR 108/78, GRUR 1979, 732, 733 - Fußballtor; OLG Köln, Urt. v. 6.3.2014, 15 U 133/13, ZUM-RD 2015, 521, 523 - Unzulässige Doppelgängerwerbung; Götting in Schricker/Loewenheim, Urheberrecht, 5. Aufl. 2017, § 22 KUG Rn. 16 ff.

5 BVerfG, Beschl. v. 14.7.2004, 1 BvR 263/03, ZUM-RD 2004, 573, 574.

6 Götting in ders./Schertz/Seitz, Handbuch des Persönlichkeitsrechts, 2008, § 2 Rn. $1 \mathrm{ff}$. 
i.V.m. Art. 2 Abs. 1, Art. 1 Abs. 1 GG nur als Rahmenrecht geschützt, das auf Grund seiner generalklauselartigen Weite und Unbestimmtheit eine umfassende Abwägung des Rechts auf informationelle Selbstbestimmung und der Kommunikationsfreiheiten im jeweiligen Einzelfall erfordert. Im Bildnisschutz wird zwar durch die durch den einfachen Gesetzgeber in $\S \S 23,24$ KUG festgelegten typisierenden Fallgruppen eine Vorrangrelation bestimmter Abwägungstopoi bestimmt, z.B. der zeitgeschichtlichen Bedeutung eines Bildnisses oder der Teilnahme einer Person an einer Versammlung. Auch hier gilt aber, dass bei der Ausfüllung der unbestimmten Tatbestandsmerkmale, wie z.B. dem Begriff der „Zeitgeschichte“ gemäß $§ 23$ Abs. 1 Nr. 1 KUG oder dem des „,berechtigten Interesses“ gemäß $§ 23$ Abs. 2 KUG, eine Abwägung der abstrakt gleichrangigen Rechtsgüter und Interessen im Einzelfall erforderlich ist. ${ }^{7}$

Die Strukturen des deutschen Datenschutzrechts wurden hingegen grundlegend dadurch geprägt, dass zunächst nicht die von privaten Akteuren, sondern die von öffentlichen Stellen verursachten Risiken für den Schutz persönlicher Daten im Vordergrund standen. Datenverarbeitungen durch die öffentliche Hand bedürfen für ihre Rechtmäßigkeit einer detaillierten gesetzlichen Ermächtigung, während privaten Akteuren aus verfassungsrechtlicher Perspektive im Ausgangspunkt eine Datenverarbeitung grundsätzlich gestattet ist, sofern sie nicht - wenn dies zum Schutze der Betroffenen erforderlich ist - untersagt ist. ${ }^{8} \mathrm{Da}$ aufgrund seiner Entstehungsgeschichte die Abwehrfunktion gegenüber dem staatlichen Handeln im Vordergrund stand, gilt im Datenschutzrecht sowohl für Datenverarbeitungen durch öffentliche Stellen als auch durch nicht-öffentliche Stellen das Regelungsmodell des Verbots mit Erlaubnisvorbehalt (s. nun Art. 6 DSGVO). Allerdings hat die DSGVO im Hinblick auf Datenverarbeitungen durch nichtöffentliche Stellen nun zu einer Annäherung der Regelungssystematiken des Äußerungs- und des Datenschutzrechts geführt, indem Art. 6 Abs. 1 lit. f) DSGVO nur die Erforderlichkeit der Datenverarbeitung zur Wahrung der berechtigten Interessen des Datenverarbeiters sowie eine Abwägung zwischen den Interessen des Betroffenen und des Datenverarbeiters fordert. Insofern ist der neue Regelungsrahmen wesentlich flexibler als die für Datenverarbeitungen durch nicht-öffentliche Stellen einschlägigen, abschließenden Erlaubnistatbestände der $\S \S 28$ ff. BDSG a.F., die nicht auf an die Öffentlichkeit gerichtete Publikationsvorgänge zugeschnitten waren. ${ }^{9}$

Auch hinsichtlich der Einwilligung als zentralem Erlaubnistatbestand bestehen gravierende Unterschiede. Zwar verlangen sowohl das Äußerungsrecht als auch das Datenschutzrecht eine informierte Einwilligung; allerdings sind die Vorgaben der DSGVO wesentlich

7 Grundlegend BGH, Urt. v. 6.3.2007, VI ZR 51/06, GRUR 2007, 527 Rn. 9 ff. - Winterurlaub; BVerfG, Beschl. v. 26.2.2008, 1 BvR 1626/07, GRUR 2008, 539 - Caroline von Hannover; EGMR (GK, Große Kammer), Urt. v. 7.2.2012, 40660/08, GRUR 2012, 745 Rn. 114 ff. - von Hannover/ Deutschland Nr. 2; s. auch BGH, Urt. v. 29.5.2018, VI ZR 56/17, GRUR 2018, 964 Rn. 9 - Tochter von Prinzessin Madeleine.

8 Bäcker, Der Staat 51 (2012), S. 91, 99 f.; Masing, NJW 2012, 2305, 2306 f.

9 Dazu Lauber-Rönsberg/Hartlaub NJW 2017, 1057, 1059; Lauber-Rönsberg ZD 2014, 177, 178. 
detaillierter. ${ }^{10}$ Zudem ist die Einwilligung im Datenschutzrecht anders als im Äußerungsrecht $^{11}$ gemäß Art. 7 Abs. 3 DSGVO grundsätzlich frei widerruflich. ${ }^{12}$

Weiterhin unterscheiden sich das Datenschutzrecht und das Äußerungsrecht dadurch, dass der Regelungsanspruch des Datenschutzrechts umfassender ist, indem es über die Zulässigkeit der Datenverarbeitung hinaus zahlreiche flankierende Regelungen trifft. Dies gilt z.B. für die Datenverarbeitungsgrundsätze in Art. 5 DSGVO sowie die Verpflichtung eines Datenschutzes durch Technikgestaltung und durch datenschutzfreundliche Voreinstellungen gemäß Art. 25 DSGVO. Auch die umfangreichen Regelungen der Betroffenenrechte in Art. 12 ff. DSGVO, z.B. Informations-, Auskunfts- und Löschungspflichten, finden in dieser Detailliertheit keine Entsprechung im Äußerungsrecht.

Grundlegende Unterschiede zwischen Datenschutz- und Äußerungsrecht bestehen des Weiteren hinsichtlich der Sanktions- und Durchsetzungsmechanismen. Das Datenschutzrecht sieht nicht nur Rechtsbehelfe der betroffenen Person gegen den Datenverarbeiter vor, z.B. Schadensersatzansprüche gemäß Art. 82 DSGVO und Unterlassungsansprüche gemäß $\S 1004$ BGB i.V.m. § 823 Abs. 1 BGB i.V.m. den datenschutzrechtlichen Normen. Zudem besteht auch eine behördliche Aufsicht (Art. 51 ff. DSGVO). Die unabhängigen Aufsichtsbehörden werden zur Erfüllung ihrer Aufgaben mit Untersuchungs- und Abhilfebefugnissen gemäß Art. 58 Abs. 1 und 2 DSGVO, die u.a. anlasslose ${ }^{13}$ Zugangsrechte zu Räumlichkeiten von Datenverarbeitern umfassen, sowie mit der Befugnis zur Verhängung von erheblichen Bußgeldern von bis zu 20 Mio. Euro oder im Fall eines Unternehmens von bis zu $4 \%$ seines gesamten weltweit erzielten Jahresumsatzes (Art. 83 DSGVO) ausgestattet. Diese Form der behördlichen Aufsicht wäre, wenn sie auch für die journalistische Arbeit gelten würde, mit der Vorstellung eines modernen, freiheitlichen Presserechts schwer vereinbar. ${ }^{14}$ Gleiches gilt für die durch Art. 5 Abs. 3 GG, Art. 10 EMRK, Art. 13 S. 1 GRCh garantierte

10 S. z.B. Art. 4 Nr. 11, Art. 7 und Erw. 32 DSGVO; s. auch KG ZD 2018, 118 Rn. 62 u. 85 zu § 4a BDSG a.F. hinsichtlich des sog. „App-Zentrums“ von Facebook.

11 S. im Hinblick auf eine nach $\S 22$ KUG erteilte Einwilligung BAG NJW 2015, 2140 Rn. 38; Götting in Schricker/Loewenheim, Urheberrecht, 5. Aufl. 2017, § 22 KUG Rn. 42.

12 Fraglich ist allerdings, ob die auch freie Widerrufbarkeit auch dann Bestand hat, wenn eine Person für die Einräumung von Nutzungsrechten ein Entgelt erhalten hat, z.B. bei Modell-Verträgen, oder ob hier ggf. auf Grundlage des venire-contra-factum-proprium-Einwandes Einschränkungen hinzunehmen sind. S. zur alten Rechtslage z.B. Gola/Klug/Körffer, in: Gola/Schomerus, Bundesdatenschutzgesetz, 12. Aufl. 2015, § 4a BDSG a.F., Rn. 37 ff. Es ist unklar, ob diese Einschränkungen auch im Rahmen des Art. 7 Abs. 3 DSGVO Bestand haben, Schulz, in: Gola, Datenschutz-Grundverordnung, 2. Aufl. 2018, Art. 7 Rn. 57; Buchner/Kühling, in: Kühling/Buchner, DS-GVO BDSG, 2. Auflage 2018, Art. 7 Rn. 38.

13 Eichler, in: BeckOK DatenschutzR, DS-GVO, 25. Edition, Stand: 1.8.2018, Art. 58 Rn. 17.

14 So zu Recht Cornils, Das datenschutzrechtliche Medienprivileg unter Behördenaufsicht?, 2018, S. 22; Veil, NVwZ 2018, 686, 689 f. S. auch Oster, in: Hartstein/Ring/u.a., RStV/JMStV, Stand: August 2018, § 9c RstV Rn. 64 ff. hinsichtlich des Rundfunks. 
Freiheit der Kunst. ${ }^{15}$ Darüber hinaus können die erheblichen Bußgeldandrohungen für Verstöße gegen datenschutzrechtliche Vorgaben sog. „chilling effects“ haben und hierdurch zu Einschränkungen der Meinungs-, Presse- und Kunstfreiheit führen, ${ }^{16}$ wenn sie auch für die journalistische oder künstlerische Arbeit gelten würden.

Wie diese kurze Gegenüberstellung zeigt, bestehen erhebliche Unterschiede zwischen dem Datenschutz- und dem Äußerungsrecht. Eine uneingeschränkte Anwendung des Datenschutzrechts auf mediale Datenverarbeitungen würde die Kommunikationsfreiheiten sowie die Kunstfreiheit stark einschränken. ${ }^{17}$ Daher ist verfassungsrechtlich eine Anpassung der datenschutzrechtlichen Vorgaben an die Funktionsbedingungen der Kommunikationsfreiheiten und der Kunstfreiheit erforderlich. ${ }^{18}$

Grundsätzlich kann dies zum einen durch entsprechende Einschränkungen innerhalb des Datenschutzrechts i.e.S. geschehen; als Beispiel ist Art. 17 Abs. 3 lit. a) DSGVO zu nennen. Zum anderen kommt alternativ eine Bereichsausnahme für von den Kommunikationsfreiheiten bzw. der Kunstfreiheit geschützte Kommunikationsvorgänge in Betracht. Diese Option hat der europäische Gesetzgeber den Mitgliedstaaten durch die Öffnungsklausel(n) des Art. 85 DSGVO $^{19}$ eröffnet, um das Recht auf den Schutz personenbezogener Daten mit den Kommunikationsfreiheiten in Einklang zu bringen.

\section{Rechtslage vor der DSGVO}

Um einen Ausgleich zwischen dem Recht auf informationelle Selbstbestimmung und den Kommunikationsfreiheiten zu schaffen, sahen bereits die Datenschutz-RL 95/46 ${ }^{20}$ und die deutschen Datenschutzgesetze auf Bundes- und Landesebene - wie nun auch die DSGVO spezielle Regelungen für Datenverarbeitungen durch die Medien vor.

15 BGH, Urt. v. 24.11.2009, VI ZR 219/08, GRUR 2010, 171 Rn. 13 - Esra: „Staatliche Maßnahmen dürfen nicht zu einer Einschüchterung des Künstlers und des für die Darbietung und Verbreitung des Kunstwerks Verantwortlichen führen." S. auch die zustimmende Anmerkung von Ladeur, NJW 2010,765 .

16 S. generell zu der Problematik der „,chilling effects“ BVerfG, Beschl. v. 10.11.1998, 1 BvR 153196, NJW 1999, 1322; BVerfG, Beschl. v. 25.6.2009, 1 BvR 134/03, NJW-RR 2010, 470 Rn. 62; Grabenwarter, in: Maunz/Dürig/u.a., GG, 83. EL April 2018, Art. 5 Abs. 1 GG Rn. 103; zu der einschüchternden Wirkung (zivilrechtlicher) Sanktionen im Falle von Persönlichkeitsrechtsverletzungen durch künstlerisches Schaffen BGH Urt. v. 24.11.2009, VI ZR 219/08, GRUR 2010, 171 Rn. 13 f. - Esra mwN; LG Berlin, Urt. v. 3.6.2014, 27 O 56/14, ZUM 2014, 729, 732; im Falle von Persönlichkeitsrechtsverletzungen durch Medien OLG Hamburg, Urt. v. 21.10.2008, 7 U 11/08, ZUM 2009, 65, 68 .

17 Pötters, in: Gola, Datenschutz-Grundverordnung, 2. Aufl. 2018, Art. 85 Rn. 4.

18 Cornils, Das datenschutzrechtliche Medienprivileg unter Behördenaufsicht?, 2018, S. 23.

19 Dazu s.u. IV.3.

20 Richtlinie 95/46/EG des Europäischen Parlaments und des Rates vom 24.10.1995 zum Schutz natürlicher Personen bei der Verarbeitung personenbezogener Daten und zum freien Datenverkehr, AB1. I 281 v. 23.11.1995. 


\section{Art. 9 Datenschutz-RL 95/46}

Art. 9 Datenschutz-RL 95/46 ${ }^{21}$ verpflichtete die Mitgliedstaaten, für die Verarbeitung personenbezogener Daten allein zu journalistischen, künstlerischen oder literarischen Zwecken von den Vorgaben der Datenschutz-Richtlinie abweichende Regelungen zu treffen, um das Recht auf Privatsphäre mit den für die Freiheit der Meinungsäußerung geltenden Vorschriften in Einklang zu bringen.

Die Freistellung der Kommunikation zu journalistischen Zwecken galt nach der Rechtsprechung des EuGH ,nicht nur für Medienunternehmen, sondern für jeden, der journalistisch tätig ist“".22 Auch den Begriff der journalistischen Tätigkeit legte der EuGH weit aus; eine Tätigkeit sei als journalistisch anzusehen, „wenn sie zum Zweck habe, Informationen, Meinungen oder Ideen, mit welchem Übertragungsmittel auch immer, in der Öffentlichkeit zu verbreiten“. So konnte nach Ansicht des EuGH auch die Publikation von durch die finnischen Steuerbehörden veröffentlichten Steuerdaten durch eine Zeitschrift ohne weitere Aufbereitung oder inhaltliche Auswertung als journalistische Tätigkeit angesehen werden. ${ }^{23}$ Dagegen fielen Suchmaschinen-Betreiber nicht unter Art. 9 Datenschutz-RL 95/46, wie der EuGH in seiner Google Spain-Entscheidung festhielt. ${ }^{24}$

Neben journalistischen Zwecken privilegierte die Regelung ebenso wie der erläuternde Erw. 37 explizit auch künstlerische Zwecke. Der Erwägungsgrund verweist zudem auf den grundrechtlichen Schutz durch Art. 10 EMRK, der die Kunstfreiheit zwar nicht ausdrücklich erwähnt, jedoch auch einen Schutz für Kommunikationsvorgänge im Rahmen des künstlerischen Schaffens vermittelt. ${ }^{25}$

\section{Deutsche Rechtslage}

Unter den früheren Fassungen des BDSG kam es aufgrund seines begrenzten Anwendungsbereichs nur in Ausnahmefällen zu einer Kollision mit den Regelungen des KUG. Daher ist

21 Die Regelung lautete: Art. 9 Verarbeitung personenbezogener Daten und Meinungsfreiheit: Die Mitgliedstaaten sehen für die Verarbeitung personenbezogener Daten, die allein zu journalistischen, künstlerischen oder literarischen Zwecken erfolgt, Abweichungen und Ausnahmen von diesem Kapitel [d.h. Kap. II: Allgemeine Bedingungen für die Rechtmäßigkeit der Verarbeitung personenbezogener Daten] sowie von den Kapiteln IV [Übermittlung personenbezogener Daten in Drittländer] und VI [Kontrollstelle und Gruppe für den Schutz von Personen bei der Verarbeitung personenbezogener Daten] nur insofern vor, als sich dies als notwendig erweist, um das Recht auf Privatsphäre mit den für die Freiheit der Meinungsäußerung geltenden Vorschriften in Einklang zu bringen. (Erläuterungen in eckigen Klammern von der Verf. hinzugefügt).

22 EuGH, Urt. v. 16.12.2008, Rs. C-73/07, MMR 2009, 175, 177 Rn. 58 - Satamedia.

23 EuGH, Urt. v. 16.12.2008, Rs. C-73/07, MMR 2009, 175, 177 Rn. 61 - Satamedia.

24 EuGH (GK), Urt. v. 13.5.2014, C-131/12, GRUR 2014, 895 Rn. 85 - Google Spain u. Google/ AEPD u. Costeja González.

25 EGMR Urt. v. 24. 5. 1988, Nr. 10737/84, Ziff. 33 - Müller; EGMR Urt. v. 25. 1. 2007, Nr. 68354/01, Ziff. 26 f. - Vereinigung Bildender Künstler/Österreich; Grabenwarter/Pabel, EMRK, § 23 Rn. 1. 
es nicht erstaunlich, dass die Problematik des Verhältnisses zwischen diesen beiden Regelungsbereichen erst in den letzten Jahren virulent geworden ist. Denn zunächst war der Anwendungsbereich des BDSG für nicht-öffentliche Stellen nur dann eröffnet, wenn eine Datenverarbeitung mit Dateibezug vorlag, d.h. in einer nach bestimmten Merkmalen zugänglichen und automatisiert oder nicht-automatisiert auswertbaren Sammlung ( 33 Abs. 2 BDSG). ${ }^{26}$ Diese Voraussetzungen waren z.B. bei einem Stapel Papierabzüge von Fotografien ohne Textbeschriftung nicht gegeben. ${ }^{27}$

Zudem unterfielen Akten, wozu zunächst auch Bild- und Tonträger zählten, nicht dem Dateibegriff, sofern sie nicht durch automatisierte Verfahren umgeordnet und ausgewertet werden konnten, ${ }^{28}$ was vor der flächendeckenden Verbreitung digitaler Aufzeichnungsgeräte verhältnismäßig selten der Fall gewesen sein dürfte. Erst seit dem BDSG 2001 gelten für Bild- und Tonträger die gleichen Grundsätze wie für andere Arten personenbezogener Daten. ${ }^{29}$ Mit der Ausdehnung des Anwendungsbereichs durch die Novellierung 2001 hatte zugleich die Subsidiaritätsklausel des $\S 1$ Abs. 3 S. 1 BDSG a.F. an praktischer Bedeutung gewonnen, da es vermehrt zu Überschneidungen mit anderen gesetzlichen Regelungen kam. ${ }^{30}$ Insofern regelte $\S 1$ Abs. 3 S. 1 BDSG a.F. als Kollisionsnorm das Verhältnis zwischen Äußerungs- und Datenschutzrecht.

Zudem sahen die deutschen Medien- und Datenschutzgesetze zur Umsetzung von Art. 9 Datenschutz-RL 95/46 mit dem sog. Medienprivileg spezielle Konkurrenzregeln für journalistische Verarbeitungen vor. ${ }^{31}$

\section{a) Medienprivileg}

Die nationalen Datenschutz- bzw. Pressegesetze befreiten die Datenverarbeitung durch Unternehmen und Hilfsunternehmen der Presse zu eigenen journalistisch-redaktionellen oder literarischen Zwecken weitgehend von den datenschutzrechtlichen Vorgaben. Soweit Presseveröffentlichungen oder Telemedien journalistisch-redaktionellen oder literarischen Zwecken dienten, wurde die in diesem Rahmen erforderliche Datenverarbeitung durch $\S 41$ Abs. 1 BDSG a.F., durch die entsprechenden Regelungen in den Landespressegesetzen so-

26 S. § 1 Abs. 2, § 2 Abs. 3 Nr. 3 BDSG 1977; § 1 Abs. 2 Nr. 3, § 3 Abs. 2 BDSG 1990.

27 S. z.B. zu $\S 3$ Abs. 2 S. 2 BDSG 2003 Simitis/Dammann, Bundesdatenschutzgesetz, 7. Aufl. 2011, BDSG § 3 Rn. 99.

28 Vgl. § 2 Abs. 3 Nr. 3 BDSG 1977; § 3 Abs. 2 und Abs. 3 BDSG 1990.

29 Im nicht-öffentlichen Bereich galt das BDSG gemäß § 1 Abs. 2 Nr. 3 BDSG 2001 für die Verarbeitung, Nutzung oder Erhebung von Daten unter Einsatz von Datenverarbeitungsanlagen sowie von Daten in oder aus nicht automatisierten Dateien. S. näher Gola/Körffer/Klug, in: Gola/Schomerus, Bundesdatenschutzgesetz, 12. Aufl. 2015, §3 Rn. 21. Hierunter fiel z.B. auch das Bereitstellen personenbezogener Daten auf eine Internetseite, EuGH, Urt. v. 6.11.2003, C-101/01, EuZW 2004, 245 (247) Rn. 25 ff. - Lindqvist zu Art. 3 Abs. 2 der Richtlinie 95/46.

30 Dix, in: Simitis, Bundesdatenschutzgesetz, 8. Aufl. 2014, § 1 BDSG Rn. 159.

31 Der Beitrag beschränkt sich auf die Darstellung der für die Presse geltenden Regelungen und geht nicht auf die Vorgaben des Rundfunkrechts ein. 
wie durch $\S 57$ Abs. 1 RStV von der Anwendbarkeit des BDSG a.F. - mit Ausnahme der $\S \S 5,9,38$ a BDSG a.F. und hierauf bezogen $\S 7$ BDSG a.F. - freigestellt.

Anders als die Bezeichnung als Medienprivileg suggeriert, handelt es sich um eine ,jedenfalls im Grundsatz verfassungsrechtlich gebotene Immunisierung “. ${ }^{32}$ Auch das BVerwG und der BGH erkannten in ihrer Rechtsprechung an, dass eine solche Freistellung gemäß Art. 5 Abs. 1 GG, Art. 10 Abs. 1 S. 2 EMRK, Art. 11 Abs. 1 S. 1 GRCh zum Schutz der für ein demokratisches Gemeinwesen konstitutiven Medienfreiheit erforderlich war. ${ }^{33}$

\section{aa) Tatbestandsvoraussetzungen}

Die Regelungen erfassten tatbestandlich die Verarbeitung und Nutzung personenbezogener Daten durch Unternehmen und Hilfsunternehmen der Presse ausschließlich zu eigenen journalistisch-redaktionellen oder literarischen Zwecken.

Begünstigte waren nicht nur Presseunternehmen im institutionellen Sinne, sondern auch selbständige Journalisten, die nicht in redaktionelle Strukturen eingebunden sind. ${ }^{34} \mathrm{Im}$ Übrigen folgte zumindest das BVerwG allerdings einem eher formalen Verständnis und sah die Veröffentlichungen einer kommunalen Wählervereinigung in der Form eines e.V. nicht als journalistische Tätigkeit an. Kunden-, Werks-, Partei- und Vereinspublikationen seien nur dann privilegiert, wenn die für die Publikationen zuständige Abteilung eine organisatorisch selbstständige, in sich geschlossene, gegenüber den sonstigen (betrieblichen) Stellen abgeschottete, in der redaktionellen Tätigkeit autonome Organisationseinheit war. ${ }^{35}$

Eine journalistisch-redaktionelle Tätigkeit lag nach Ansicht des BGH vor, wenn die Absicht zu einer an die Allgemeinheit gerichteten Berichterstattung i.S. des Art. 5 Abs. 1 S. 2 GG bestand. Das datenschutzrechtliche Medienprivileg sah der Gerichtshof als einschlägig an, wenn die Medien ihrem verfassungsrechtlichen Auftrag gemäß zur Information der Öffentlichkeit und zur Mitwirkung an der demokratischen Willensbildung beitrügen. ${ }^{36}$ Unter diesen Umständen waren die Recherche, Redaktion, Veröffentlichung, Dokumentation und Archivierung personenbezogener Daten zu publizistischen Zwecken umfassend ge-

32 Cornils, Das datenschutzrechtliche Medienprivileg unter Behördenaufsicht?, 2018, S. 23. Zur Kritik an dem Begriff des Medienprivilegs s. auch Westphal, in: Taeger/Gabel, BDSG, 2. Aufl. 2013, $\S 41$ Rn. 15; Dix, in: Simitis, Bundesdatenschutzgesetz, 8. Aufl. 2014, § 41 Rn. 1; Thomale, Die Privilegierung der Medien im deutschen Datenschutzrecht, 2007, S. 3.

33 BVerwG, Beschl. v. 29.10.2015, 1 B 32.15, ZD 2016, 193 Rn. 5; BGH, Urt. v. 9.2.2010, VI ZR 243/08, GRUR 2010, 549, 552 Rn. 26 - Spiegel-Dossier.

34 BVerwG, Beschl. v. 29.10.2015, 1 B 32.15, ZD 2016, 193 Rn. 5; Dix, in: Simitis, Bundesdatenschutzgesetz, 8. Aufl. 2014, § 41 Rn. 11; Kamp, Personenbewertungsportale, 2011, S. 161; Thomale, Die Privilegierung der Medien im deutschen Datenschutzrecht, 2007, S. 76; Weichert, VuR 2009, 323, 324.

35 BVerwG, Beschl. v. 29.10.2015, 1 B 32.15, ZD 2016, 193 Rn. 4 f.

36 BGH, Urt. v. 9.2.2010, VI ZR 243/08, GRUR 2010, 549, 552 f. Rn. 28 ff. - Spiegel-Dossier; s. auch BGH, Urt. v. 15.12.2009, VI ZR 227/08, NJW 2010, 757, 760 Rn. 25 - Sedlmayr-dradio.de zu $§ 17$ Abs. 1 Deutschlandradio-Staatsvertrag. 
schützt, nicht hingegen hiervon unabhängige Datenverarbeitungen durch Medien wie z.B. die Personaldatenverarbeitung oder Datenverarbeitungen im Zusammenhang mit dem Gebühreneinzug. ${ }^{37}$ Unter das Medienprivileg fiel auch die Bereitstellung von Beiträgen im Rahmen eines Online-Archivs eines Presseunternehmens. ${ }^{38}$

Aufgrund der inkonsistenten Rechtsprechung des BGH war jedoch unklar, unter welchen Voraussetzungen das Medienprivileg für Beiträge durch Informationsintermediäre galt. Einerseits führte der BGH in Bezug auf Personenbewertungsportale aus, dass hier in der Regel keine Datenverarbeitung für journalistisch-redaktionelle Zwecke erfolge. Diese setzte voraus, dass die meinungsbildende Wirkung für die Allgemeinheit prägender Bestandteil des Angebots und nicht nur schmückendes Beiwerk sei. Dies sei bei einer bloß automatischen Auflistung von redaktionellen Beiträgen und der Errechnung von Durchschnittsnoten durch Personenbewertungsportale nicht gegeben. ${ }^{39}$ Andererseits hat der BGH in Bezug auf andere Intermediäre diese Linie nicht fortgesetzt, sondern im Hinblick auf Suchmaschinen- ${ }^{40}$ und Blogbetreiber ${ }^{41}$ nur äußerungsrechtliche Ansprüche geprüft und die Anwendbarkeit des Datenschutzrechts nicht einmal diskutiert. ${ }^{42}$ Dabei hätte es bei konsequenter Fortführung der zu den Personenbewertungsportalen entwickelten Rechtsprechung nahegelegen, auch in der Blog-Eintrag-Entscheidung die Privilegierung des $§ 41$ BDSG zu verneinen und folglich die datenschutzrechtlichen Regelungen anzuwenden, da der journalistische Eigenanteil des Blogbetreibers zumindest ebenso gering war wie derjenige des Personenbewertungsportalbetreibers. ${ }^{43}$ Auch in jüngeren Entscheidungen zu Personenbewertungsportalen stützte sich der BGH wiederum nur auf das Äußerungsrecht. ${ }^{44}$

37 BGH, Urt. v. 9.2.2010, VI ZR 243/08, GRUR 2010, 549 Rn. 28 - Spiegel-Dossier.

38 BGH, Urt. v. 9.2.2010, VI ZR 243/08, GRUR 2010, 549 Rn. 30 - Spiegel-Dossier.

39 BGH, Urt. v. 23.6.2009, VI ZR 196/08, NJW 2009, 2888, 2890 Rn. 21 f. - spickmich.de; BGH, Urt. v. 23.9.2014, VI ZR 358/13, NJW 2015, 489 Rn. 13 - Ärztebewertung II; BGH, Urt. v. 20.2.2018, VI ZR 30/17, GRUR 2018, 636 Rn. 10 - Ärztebewertung III; OVG Münster Urt. v. 19.10.2017, Az. 16 A 770/17, NVwZ 2018, 742 Rn. 50 - Fahrerbewertungsportal. Anders liegt der Fall aber dann, wenn der Plattformbetreiber eine inhaltlich-redaktionelle Kontrolle ausübt oder die nutzergenerierten Inhalte durch suggestive Fragestellungen oder Kategorien im Rahmen von Eingabemasken mitprägt, was auch zu einer haftungsrechtlichen Verantwortlichkeit führen würde, s. BGH, Urt. v. 4.4.2017, VI ZR 123/16, GRUR 2017, 844 Rn. 18 - klinikbewertungen.de; BGH, Urt. v. 12.11.2009, I ZR 166/07, GRUR 2010, 616 Rn. 25 f. - marions-kochbuch.de; Spindler, Persönlichkeitsschutz im Internet, Gutachten F zum 69. Deutschen Juristentag, 2012, S. 61 ff.

40 BGH, Urt. v. 14.5.2013, VI ZR 269/12, ZUM 2013, 550 - Autocomplete-Funktion; anders dagegen OLG Hamburg, Beschl. v. 13.11.2009, 7 W 125/09, ZUM-RD 2010, 74, 75 f.: Zulässigkeit der Übermittlung von Suchergebnissen gem. § 29 Abs. 1 Nr. 2 BDSG, aber offengelassen, ob eine Datenverarbeitung oder -nutzung i.S. des BDSG vorliegt.

41 BGH, Urt. v. 25.10.2011, VI ZR 93/10, GRUR 2012, 311 - Blog-Eintrag.

42 S. z.B. BGH, Urt. v. 27.3.2012, VI ZR 144/11, GRUR 2012, 751 - RSS-Feeds; LG Tübingen, Urt. v. 18.7.2012, 7 O 525/10, ZUM-RD 2013, 345 - Nennung persönlicher Daten in Wikipedia.

43 Dazu s. näher Lauber-Rönsberg ZD 2014, 182.

44 BGH, Urt. v. 20.2.2018, VI ZR 30/17, GRUR 2018, 636 - Ärztebewertungsportal III. 
Des Weiteren privilegierten die Regelungen auch literarische Zwecke, nicht hingegen im Gegensatz zur heutigen Rechtslage ${ }^{45}$ - Datenverarbeitungen für sonstige künstlerische Zwecke. Der Begriff des literarischen Zwecks wurde weit ausgelegt und umfasste wissenschaftliche Literatur ebenso wie Unterhaltungsliteratur. ${ }^{46}$ Im Einklang mit dieser Regelung hat die Rechtsprechung die rechtlichen Vorgaben für die Verarbeitung von realen Personen als Vorbilder für Romanfiguren nicht aus dem Datenschutzrecht, sondern allein aus dem Äußerungsrecht abgeleitet, allerdings ohne dessen Verhältnis zum Datenschutzrecht und die Einschlägigkeit des Medienprivilegs näher zu thematisieren. ${ }^{47}$ Dies erklärt sich wohl auch aus der Entstehungsgeschichte der Normen: Als die ersten Datenschutzgesetze Ende der 1970er Jahre in Kraft traten, bestand bereits eine etablierte Rechtsprechung für den Ausgleich zwischen der Kunstfreiheit sowie dem durch den BGH in der grundlegenden Leserbrief-Entscheidung ${ }^{48}$ aus dem Jahr 1954 anerkannten zivilrechtlichen allgemeinen Persönlichkeitsrecht. ${ }^{49}$

\section{bb) Äußerungsrecht als ,Lü̈ckenfüller“}

Die Freistellung von Datenverarbeitungen für journalistische und literarische Zwecke von den datenschutzrechtlichen Vorgaben wurde insbesondere im datenschutzrechtlichen Schrifttum scharf kritisiert. ${ }^{50}$ Diese Kritik verkannte jedoch, dass trotz dieser Beschränkung kein rechtliches Vakuum entstand, da - neben der als alleiniges Regelungsinstrument wohl tatsächlich unzureichenden freiwilligen Selbstregulierung im Printbereich durch den Pressekodex ${ }^{51}$ und durch die Beschwerdeordnung des Deutschen Presserates ${ }^{52}$ - die Regelungen des zivilrechtlichen Äußerungsrechts, z.B. das KUG und der Schutz des allgemeinen Persönlichkeitsrechts im Rahmen von $\S 823$ Abs. 1 BGB i.V.m. Art. 2 Abs. 1, Art. 1 GG anwendbar blieben, die sich durch ihre Flexibilität und Auslegungsoffenheit als besser geeignet erwiesen haben, um dem Persönlichkeitsschutz im Spannungsverhältnis mit der grundrechtlich geschützten Medienberichterstattung Rechnung zu tragen. ${ }^{53}$

45 S. dazu u. IV.3.a) (2).

46 Buchner, in: BeckOK DatenschutzR, 25. Ed. 1.2.2018, § 41 BDSG a.F. Rn. 32.

47 BGH, Urt. v. 21.6.2005, VI ZR 122/04, GRUR 2005, 788 - Esra; BGH, Urt. v. 24.11.2009, VI ZR 219/08, GRUR 2010, 171 Rn. 13 - Esra.

48 BGH, Urt. v. 25.5.1954, I ZR 211/53, NJW 1954, 1404 - Leserbrief.

49 S. z.B. BGH, Urt. 8.6.1982, VI ZR 139/80, NJW 1983, 1194.

50 Westphal, in: Taeger/Gabel, BDSG, 2. Aufl. 2013, §41 Rn. 32; zur neuen Rechtslage s. auch Buchner/Tinnefeld, in: Kühling/Buchner, DS-GVO BDSG, 2. Auflage 2018, Art. 85 Rn. 32.

51 Vgl. Ziff. 8 und die dazu erlassenen Richtlinien, insbes. 8.8.

52 S. $\S 1$ Abs. 1 S. $2, \S 3$ Abs. 2. Kritisch aber zu ihrer Effektivität Buchner/Tinnefeld, in: Kühling/ Buchner, DS-GVO BDSG, 2. Auflage 2018, Art. 85 Rn. 32.

53 S. auch Cornils, Das datenschutzrechtliche Medienprivileg unter Behördenaufsicht?, 2018, S. 23 ff. 


\section{b) Subsidiarität des BDSG gegenüber dem Äußerungsrecht}

Für Fallkonstellationen, die nicht unter das Medienprivileg fielen, hatte die Subsidiaritätsklausel des $\S 1$ Abs. 3 S. 1 BDSG a.F. eine nicht zu unterschätzende Bedeutung für die Auflösung der Kollision zwischen dem Datenschutzrecht und dem Äußerungsrecht. Gemäß $\S 1$ Abs. 3 S. 1 BDSG a.F. kam anderen Rechtsvorschriften des Bundes, die auf personenbezogene Daten einschließlich deren Veröffentlichung anzuwenden waren, Vorrang vor den Vorschriften BDSG zu.

Das BAG ordnete das KUG explizit als bereichsspezifische, spezialgesetzliche Regelung ein. ${ }^{54}$ Auch die zivilgerichtliche Rechtsprechung ging in der Regel im Ergebnis von einem Vorrang des KUG aus, ohne das Verhältnis zwischen dem KUG und dem BDSG zu erörtern. So wendeten die Zivilgerichte bei der Veröffentlichung von Bildnissen im Internet durch Unternehmen z.B. zu Marketingzwecken sowie durch Privatpersonen in Ausübung ihrer Meinungsfreiheit nicht das BDSG, sondern das KUG an. ${ }^{55}$ Das Gleiche gilt - mit Ausnahme der Entscheidungen zu Personenbewertungsportalen ${ }^{56}$ - in Bezug auf das allgemeine Persönlichkeitsrecht. ${ }^{57}$

Auch Fallkonstellationen im Spannungsfeld zwischen der Kunstfreiheit und den Persönlichkeitsrechten, die die Bereiche der Fotografie und des Films sowie der bildenden Kunst betrafen, welche im Gegensatz zu dem Bereich der Literatur nicht von den Medienprivilegien erfasst wurden, wurden von den Zivilgerichten allein auf Grundlage des Äußerungsrechts entschieden. ${ }^{58}$ Wie die Regelung des $\S 23$ Abs. 1 Nr. 4 KUG belegt, entsprach es auch durchaus der Intention des Gesetzgebers, dieses Spannungsfeld im Äußerungsrecht zu regeln. Hiermit wurde zugleich der sich aus Art. 9 Datenschutz-RL 95/46 ergebende Regelungsauftrag erfüllt, von den Vorgaben der Richtlinie abweichende Regelungen zu treffen, um das Datenschutzrecht mit der Kunstfreiheit in Einklang zu bringen.

Ihrer Rechtsnatur entsprechend, stand es den nationalen Gesetzgebern frei, wie sie die Vorgaben der Datenschutz-RL 95/46 im nationalen Recht umsetzten. Insofern war es mit der Richtlinie vereinbar, dass der Ausgleichsmechanismus im nationalen Recht z.T. auf der Subsidiaritätsklausel des $\S 1$ Abs. 3 BDSG a.F. basierte, die dazu führte, dass bereichsspezi-

54 BAG, Urt. v. 11.12.2014, 8 AZR 1010/13, NJW 2015, 2140 Rn. 16, 25 ff. - Einwilligung des Arbeitnehmers.

55 BGH, Urt. v. 11.11.2014, VI ZR 9/14, NJW 2015, 1450 - Hostess auf Eventportal; BGH, Urt. v. 8.4.2014, VI ZR 197/13, NJW-RR 2014, 1193 - Mieterfest; OLG Karlsruhe, Beschl. V. 8.7.2016, 18 WF 183/15, NJW-RR 2016, 1158 - Veröffentlichung privater Fotos im Internet.

56 BGH, Urt. v. 23.6.2009, VI ZR 196/08, NJW 2009, 2888 - spickmich.de; BGH, Urt. v. 23.9.2014, VI ZR 358/13, NJW 2015, 489 Rn. 13 - Ärztebewertung II; BGH, Urt. v. 20.2.2018, VI ZR 30/17, GRUR 2018, 636 Rn. 10 - Ärztebewertung III.

57 Vgl. z.B. BGH, Urt. v. 5.6.2008, I ZR 96/07, NJW 2008, 3782 - Zerknitterte Zigarettenschachtel.

58 BGH, Versäumnisurt. v. 26.5.2009, VI ZR 191/08, NJW 2009, 3576 - „Kannibale von Rotenburg"; s. auch noch BGH, Urt. v. 20.7.2018, V ZR 130/17, BeckRS 2018, 22188 Rn. 22 ff. Wie dargestellt fielen zudem vor der Novellierung des BDSG im Jahr 2001 Bild- und Tonträger in der Regel nicht in den Anwendungsbereich des BDSG; dazu s.o. unter 2. 
fischen Regelungen, wie dem KUG in Bezug auf das Recht am eigenen Bild, Vorrang zukam.

\section{Rechtslage seit Anwendbarkeit der DSGVO}

Aufgrund der unmittelbaren Rechtsverbindlichkeit der DSGVO (Art. 288 Abs. 2 AEUV) und ihres Anwendungsvorrangs ${ }^{59}$ hat sich die Rechtslage nunmehr grundlegend geändert. Dem nationalen Recht verbleibt nur dann ein Anwendungsbereich neben der DSGVO, wenn deren sachlicher Anwendungsbereich eingeschränkt ist oder wenn der europäische Gesetzgeber den Mitgliedstaaten durch eine der zahlreichen expliziten oder impliziten Öffnungsklauseln die Befugnis zur Konkretisierung der DSGVO oder Abweichung von ihren Regelungen eingeräumt hat. ${ }^{60}$ Daher hat das Inkrafttreten der DSGVO nicht nur eine grundlegende Reform der nationalen Datenschutzgesetze erforderlich gemacht, sondern die gesamte Tektonik des Datenschutzrechts fundamental verändert: Die datenschutzrechtlichen Vorgaben für in den Anwendungsbereich der DSGVO fallende Datenverarbeitungen ergeben sich vorrangig aus der Verordnung und werden durch die nationalen Bestimmungen im BDSG und den bereichsspezifischen Datenschutzgesetzen ergänzt, sodass die verschiedenen Normebenen ineinander verschränkt sind. Insoweit sehen die reformierten deutschen Datenschutzgesetze auf Bundes- und Landesebene kein datenschutzrechtliches Vollregime vor. Datenschutzrecht wird hierdurch zu einer Materie der „Ko-Regulierung“ von EU und Mitgliedstaaten. ${ }^{61}$ Diese letztlich aus der unmittelbaren Anwendbarkeit der DSGVO folgende Fragmentarität des nationalen Rechts führt dazu, dass die Regelungssystematik wesentlich komplexer ist als nach der alten Rechtslage. ${ }^{62}$

Die DSGVO enthält keine mit $\S 1$ Abs. 3 BDSG a.F. vergleichbare Subsidiaritätsklausel. Sie erfasst somit grundsätzlich auch Materien, die im deutschen Recht bislang im Rahmen des Äußerungsrechts geregelt wurden. Die Subsidiaritätsklausel des $§ 1$ Abs. 2 BDSG n.F. greift nur dann ein, wenn der Anwendungsbereich des nationalen Datenschutzrechts eröffnet ist.

Für das Verhältnis zwischen Datenschutz- und Äußerungsrecht bedeutet dies, dass sich die maßgeblichen Kollisionsregeln aus der DSGVO und nicht mehr aus dem nationalen Recht ergeben.

59 Kühling/Martini et al., Die DSGVO und das nationale Recht, S. 3; Roßnagel, in: ders., Europäische Datenschutz-Grundverordnung, 2017, § 2 Rn. 2 ff.

60 Wolff, in: Schantz/Wolff, Das neue Datenschutzrecht, S. 67 ff. Dagegen besteht im Übrigen keine Befugnis der Mitgliedstaaten, die DSGVO zu präzisieren, zu konkretisieren oder zu ergänzen, ders. Schantz/Wolff, Das neue Datenschutzrecht, S. 66.

61 Roßnagel, DuD 2017, 277 (278).

62 S. auch Kühling/Raab, in: Kühling/Buchner, DS-GVO BDSG, 2. Auflage 2018, A. Einführung Rn. 129. 


\section{Einschränkung des Anwendungsbereichs bei Datenverarbeitungen für persönliche oder familiäre Tätigkeiten}

Der umfassende Anwendungsbereich der DSGVO wird allerdings durch die in Art. 2 Abs. 2 DSGVO geregelten Ausnahmen eingeschränkt. Relevant ist hier Art. 2 Abs. 2 lit.c) DSGVO, der Datenverarbeitungen durch natürliche Personen zur Ausübung ausschließlich persönlicher oder familiärer Tätigkeiten aus dem Anwendungsbereich der DSGVO ausschließt. ${ }^{63}$ Die Ausnahme des Art. 2 Abs. 2 lit. c DSGVO bezieht sich laut Erw. 18 z.B. auf privaten Schriftverkehr und private Anschriftenverzeichnisse, die nicht den datenschutzrechtlichen Vorgaben unterliegen.

Der Erwägungsgrund birgt allerdings die Gefahr von Missverständnissen, da er daneben auch „die Nutzung sozialer Netzwerke und Online-Tätigkeiten“ erwähnt; dies soll jedoch ausschließlich „,im Rahmen der genannten Tätigkeiten“ gelten, d.h. nur bei Kommunikationsvorgängen im Internet im Rahmen persönlicher oder familiärer Tätigkeiten. Die mit Datenverarbeitungen im Internet einhergehenden Risiken für den Persönlichkeitsschutz sprechen dafür, den Wortlaut des Erwägungsgrundes eng auszulegen, sodass Internetnutzungen nur dann aus dem Anwendungsbereich des Datenschutzrechts herausfallen, wenn der Kreis der Empfänger auf das persönliche oder familiäre Umfeld begrenzt ist, dagegen nicht bei privat motivierten Veröffentlichungen im Internet, die einem unbegrenzten Empfängerkreis zugänglich sind. ${ }^{64}$

Zudem kann eine Verarbeitung personenbezogener Daten im Einklang mit der bisherigen Rechtsprechung des EuGH zu Art. 3 Abs. 2 Datenschutz-RL 95/46 nur dann unter die Ausnahme fallen, wenn sie in der ausschließlich persönlichen oder familiären Sphäre desjenigen vorgenommen wird, der die Daten verarbeitet. Aus diesem Grund hat der EuGH eine durch einen Hauseigentümer an seinem Einfamilienhaus angebrachte Videoüberwachung, die sich teilweise auch auf den öffentlichen Raum erstreckte, nicht als ausschließlich persönliche oder familiäre Tätigkeit i.S. von Art. 3 Abs. 2 Datenschutz-RL 95/46 angesehen. ${ }^{65}$

Soweit eine Datenverarbeitung im Rahmen ausschließlich persönlicher oder familiärer Tätigkeiten im Sinne von Art. 2 Abs. 2 lit. c) DSGVO erfolgt, ist somit nicht der Anwendungsbereich der DSGVO, sondern der des nationalen Rechts eröffnet. Die insoweit parallele Regelung des $\S 1$ Abs. 1 S. 2 BDSG schränkt den Anwendungsbereich des BDSG ebenfalls ein. In diesen Fällen gelten daher die zivilrechtlichen Regelungen des Persönlich-

63 Vgl. den insoweit parallelen $\S 1$ Abs. 2 Nr. 3 BDSG a.F.

64 Albrecht/Jotzo, Das neue Datenschutzrecht, S. 67; Schantz NJW 2016, 1841, 1843. Ebenso zu Art. 3 Abs. 2 Datenschutz-RL 95/46 EuGH, Urt. v. 6.11.2003, C-101/01, EuZW 2004, 245, 247 Rn. 47 - Lindqvist; EuGH, Urt. v. 16.12.2008, Rs. C-73/07, MMR 2009, 175 Rn. 44 - Satamedia; s. auch EuGH, Urt. v. 10.7.2018, C-25/17, ZD 2018, 469 Rn. 42 - Zeugen Jehovas. S. zu Art. 2 Abs. 2 lit.c) DSGVO LG Frankfurt, Urt. v. 13.9.2018, 2-03 O 283/18, BeckRS 2018, 23105, Rn. 23.

65 EuGH, Urt. v. 11.12.2014, C-212/13, NJW 2015, 463 Rn. 31 ff. - Ryneš. 
keitsschutzes, wie eine nicht-veröffentlichte, intime Bild- oder Filmaufnahmen betreffende Entscheidung des BGH zeigt. ${ }^{66}$

\section{Kein postmortaler Schutz durch die DSGVO}

Darüber hinaus wird der sachliche Anwendungsbereich der DSGVO dadurch eingeschränkt, dass sie nach Erw. 27 S. 1 DSGVO nicht für personenbezogene Daten Verstorbener gilt. Zwar steht es den Mitgliedstaaten nach der Öffnungsklausel ${ }^{67}$ des Erw. 27 S. 2 DSGVO frei, Vorschriften im nationalen Recht vorzusehen. Der deutsche Gesetzgeber hat jedoch die Anwendbarkeit des Datenschutzrechts nicht auf diese Sachverhalte erstreckt. Vielmehr wird durch äußerungsrechtliche Regelungen, z.B. §22 S. 3 KUG und das auf Art. 1 Abs. 1 GG gestützte postmortale Persönlichkeitsrecht, das zum einen den Schutz des Lebensbilds ${ }^{68}$ und zum anderen den Schutz der vermögenswerten Bestandteile für eine Dauer von zehn Jahren ${ }^{69}$ beinhaltet, ein (begrenzter) postmortaler Schutz gewährleistet. ${ }^{70}$

\section{3. Öffnungsklausel(n) des Art. 85 DSGVO}

An die Stelle des Art. 9 Datenschutz-RL 95/46 ist nunmehr die Regelung des Art. 85 DSGVO getreten. Ebenso wie unter der Datenschutz-RL überantwortet der Verordnungsgesetzgeber es den Mitgliedstaaten, einen Ausgleich zwischen dem Recht auf den Schutz personenbezogener Daten einerseits und den Kommunikationsfreiheiten und der Kunstfreiheit andererseits zu schaffen. Zu diesem Zweck enthält Art. 85 Öffnungsklauseln, deren Umfang allerdings in mehrfacher Hinsicht unklar ist. Die Regelung lautet:

Artikel 85 Verarbeitung und Freiheit der Meinungsäußerung und Informationsfreiheit

(1) Die Mitgliedstaaten bringen durch Rechtsvorschriften das Recht auf den Schutz personenbezogener Daten gemäß dieser Verordnung mit dem Recht auf freie Meinungsäußerung und Informationsfreiheit, einschließlich der Verarbeitung zu journalistischen Zwecken und zu wissenschaftlichen, künstlerischen oder literarischen Zwecken, in Einklang.

(2) Für die Verarbeitung, die zu journalistischen Zwecken oder zu wissenschaftlichen, künstlerischen oder literarischen Zwecken erfolgt, sehen die Mitgliedstaaten Abweichungen oder Ausnahmen von Kapitel II (Grundsätze), Kapitel III (Rechte der betroffenen Person), Kapitel IV (Verantwortlicher und Auftragsverarbeiter), Kapitel V (Übermittlung personenbezogener Daten an Drittländer oder an internationale Organisationen), Kapitel VI (Unabhängige Aufsichtsbehörden), Kapitel VII (Zusammenarbeit und Kohärenz) und Kapitel IX (Vorschriften für besondere Verarbeitungssituationen) vor, wenn dies erforderlich ist, um das Recht auf Schutz der perso-

66 BGH, Urt. v. 13.10.2015, VI ZR 271/14, GRUR 2016, 315 Rn. 26 ff. - Intime Fotos zu § 1 Abs. 2 Nr. 3 BDSG aF.

67 Kühling/Martini et al., Die DSGVO und das nationale Recht, 21.

68 BGH, Urt. v. 20.3.1968, I ZR 44/66, NJW 1968, 1773 - Mephisto.

69 BGH, Urt. v. 5.10.2006, I ZR 277/03, GRUR 2007, 168 - kinski.klaus.

70 Dazu s. ausführlich Brändel, in: Götting/Schertz/Seitz, Handbuch des Persönlichkeitsrechts, 1. Aufl. 2008, §37 mit kritischen Anmerkungen u.a. zu Defiziten hinsichtlich der Sanktionen bei Rechtsverletzungen. 
nenbezogenen Daten mit der Freiheit der Meinungsäußerung und der Informationsfreiheit in Einklang zu bringen.

(3) Jeder Mitgliedstaat teilt der Kommission die Rechtsvorschriften, die er aufgrund von Absatz 2 erlassen hat, sowie unverzüglich alle späteren Änderungsgesetze oder Änderungen dieser Vorschriften mit.

Erw. 153 S. 5 bringt zum Ausdruck, dass der europäische Gesetzgeber es bewusst in Kauf genommen hat, dass zwischen den nationalen Rechtsordnungen Divergenzen bestehen werden. Insofern strebt die DSGVO damit in diesem Bereich keine Vollharmonisierung an, sondern räumt den Mitgliedstaaten einen eigenen Einschätzungsspielraum ein. ${ }^{71}$

Bei grenzüberschreitenden Sachverhalten stellt sich daher die Frage, welches nationale Recht Anwendung findet. Art. 3 DSGVO regelt zwar den räumlichen Anwendungsbereich der DSGVO, trifft aber keine kollisionsrechtliche Regelung. Vielmehr soll sich die räumliche Anwendbarkeit des nationalen Rechts entweder aus der Öffnungsklausel selbst ergeben oder nach Maßgabe der aufgrund der Öffnungsklausel erlassenen nationalen Regelung zu beurteilen sein, sofern diese eine Regelung zu ihrer räumlichen Anwendbarkeit trifft. ${ }^{72}$ Erw. 153 S. 5 gibt vor, dass das Recht des Mitgliedstaats angewendet werden soll, dem der Verantwortliche unterliegt. Dies wird wohl so auszulegen sein, dass nicht nur das materielle, sondern auch das Kollisionsrecht des jeweiligen Mitgliedstaats Anwendung findet, da den Erwägungsgründen, die nicht Bestandteil des Normtexts selbst sind, keine Rechtswirkung zukommt, sie also das nationale Kollisionsrecht nicht derogieren können; vielmehr sind sie nur Auslegungs- und Orientierungshilfen. ${ }^{73}$ Wenn also auf Grundlage von Art. 85 DSGVO das deutsche Recht zur Anwendung kommt und das Datenschutzrecht einschlägig sein sollte, wäre die Frage des räumlichen Anwendungsbereichs des BDSG nach § 1 Abs. 4 BDSG zu beurteilen. ${ }^{74}$ Kommt dagegen im Rahmen der Öffnungsklausel des Art. 85 DSGVO das Äußerungsrecht zur Anwendung, dann würde die Frage des anwendbaren Rechts aus deutscher Sicht durch das für unerlaubte Handlungen geltende anwendbare Kollisionsrecht des Art. 40 EGBGB bestimmt.

\section{a) Datenverarbeitungen zu journalistischen, wissenschaftlichen, künstlerischen und literarischen Zwecken, Art. 85 Abs. 2 DSGVO}

Art. 85 Abs. 2 DSGVO enthält einen konkreten Regelungsauftrag an die Mitgliedstaaten, für Datenverarbeitungen zu journalistischen, wissenschaftlichen, künstlerischen oder literarischen Zwecken Abweichungen oder Ausnahmen vorzusehen, soweit dies erforderlich ist, um das Recht auf Schutz der personenbezogenen Daten mit den Kommunikationsfreiheiten

71 Benecke/Wagner, DVB1 2016, 600 603; Cornils, Das datenschutzrechtliche Medienprivileg unter Behördenaufsicht?, 2018, S. 29; Kühling/Martini et al., Die DSGVO und das nationale Recht, 293.

72 Klar, in: Kühling/Buchner, DS-GVO BDSG, 2. Auflage 2018, Art. 3 Rn. 107 f.

73 Wegener, in Calliess/Ruffert, EUV/AEUV, Art. 288 AEUV Rn. 16.

74 Specht/Bienemann, in: Sydow, Europäische Datenschutzgrundverordnung, 2. Aufl. 2018, Art. 85 Rn. 18. 
in Einklang zu bringen. Aus Art. 85 Abs. 2 DSGVO ergibt sich somit nicht nur eine Ausgestaltungskompetenz, sondern zugleich auch eine Ausgestaltungsverpflichtung der Mitgliedstaaten. $^{75}$

\section{aa) Tatbestandsvoraussetzungen}

Die Öffnungsklausel des Art. 85 Abs. 2 DSGVO gilt für Verarbeitungen zu journalistischen Zwecken sowie zu wissenschaftlichen, künstlerischen oder literarischen Zwecken. Hierin liegt eine Erweiterung gegenüber Art. 9 Datenschutz-RL 95/46, der wissenschaftliche Verarbeitungszwecke noch nicht erwähnte. ${ }^{76}$ Zudem privilegierte die Richtlinie nur solche Verarbeitungen, die ,allein“ den genannten Zwecken dienten, während Art. 85 Abs. 2 DSGVO auch komplexere Zwecke erfasst. ${ }^{77}$

Aus dem Wortlaut von Art. 85 Abs. 2 DSGVO ergibt sich, dass sich die Öffnungsklausel auf Datenverarbeitungen zu den genannten Zwecken beschränkt. ${ }^{78}$ Für Datenverarbeitungen zu anderen Zwecken - z.B. Veröffentlichungen durch Vereine oder Unternehmen zwecks Öffentlichkeitsarbeit oder Werbung, rein privat motivierte Veröffentlichungen sowie Datenverarbeitungen durch Portalbetreiber und andere Intermediäre - kommt daher nur die Anwendung von Art. 85 Abs. 1 DSGVO in Betracht.

\section{(1) Journalistische Zwecke}

Der Begriff der journalistischen Tätigkeit ist gemäß Erw. 153 im Einklang mit der bisherigen Rechtsprechung des EuGH ${ }^{79}$ weit auszulegen, ${ }^{80}$ sodass hierunter neben Presse, Rundfunk und Telemedien auch Medienarchive fallen. Unklar ist weiterhin, unter welchen Voraussetzungen Blogger oder auch nicht-professionelle Akteure, z.B. Veröffentlichungen von Privatpersonen in ihrer Freizeit auf Weblogs oder Netzwerkplattformen, hierunter subsumiert werden können. Es spricht vieles dafür, hier darauf abzustellen, ob eine Veröffentlichung unter Wahrung gewisser Mindeststandards erfolgt mit dem Ziel, Informationen, Mei-

75 Kühling/Martini/et al., Die DSGVO und das nationale Recht, 292 f.; Albrecht/Janson, CR 2016, 500, 502; v. Lewinski, in: Auernhammer, DSGVO/BDSG, 6. Aufl. 2018, Art. 85 Rn. 21; Schiedermair, in: Ehmann/Selmayr, DS-GVO, 2. Auflage 2018, Art. 85 Rn. 23; Pötters, in: Gola, Datenschutz-Grundverordnung, 2. Aufl. 2018, Art. 85 Rn. 14; Specht/Bienemann, in: Sydow, Europäische Datenschutzgrundverordnung, 2. Auflage 2018, Art. 85 Rn. 10; Dix, in: Simitis/Hornung/ Spiecker, Datenschutzrecht, 1. Aufl. 2019, Art. 85 Rn. 26.

76 Schiedermair, in: Ehmann/Selmayr, DS-GVO, 2. Auflage 2018, Art. 85 Rn. 2.

77 Cornils, Das datenschutzrechtliche Medienprivileg unter Behördenaufsicht?, 2018, S. 30; Schiedermair, in: Ehmann/Selmayr, DS-GVO, 2. Auflage 2018, Art. 85 Rn. 24.

78 Buchner/Tinnefeld, in: Kühling/Buchner, DS-GVO, Art. 85 Rn. 14; Specht/Bienemann, in: Sydow, Europäische Datenschutzgrundverordnung, DSGVO Art. 85 Rn. 6.

79 EuGH, Urt. v. 16.12.2008, Rs. C-73/07, MMR 2009, 175, 177 Rn. 58 - Satamedia.

80 Buchner/Tinnefeld, in: Kühling/Buchner, DS-GVO, 2. Aufl. 2018, Art. 85 Rn. 17; Specht/Bienemann, in: Sydow, DSGVO, 2. Aufl. 2018, Art. 85 Rn. 14. S. o. III.1. 
nungen oder Ideen zu Angelegenheiten von öffentlichem Interesse zur öffentlichen Diskussion zu stellen. ${ }^{81}$ Die Relevanz dieser Abgrenzung hängt sehr davon ab, welche rechtlichen Rahmenbedingungen für nicht-journalistische Veröffentlichungen im Rahmen von Art. 85 Abs. 1 DSGVO anwendbar sind. ${ }^{82}$ Auf Suchmaschinen dürfte Art. 85 Abs. 2 DSGVO im Einklang mit der bisherigen Rechtsprechung des $\mathrm{EuGH}^{83}$ weiterhin nicht anwendbar sein.

Weiterhin gilt, dass Art. 85 Abs. 2 DSGVO nicht jede Datenverarbeitung erfasst, sondern nur solche, die den privilegierten Zwecken dienen. ${ }^{84}$

(2) Künstlerische und literarische Zwecke

Neben den bereits in Art. 9 Datenschutz-RL 95/46 genannten literarischen Zwecken privilegiert Art. 85 Abs. 2 DSGVO nun auch künstlerische Zwecke. Hierunter sind alle durch Art. 13 GRCh geschützten Kunstformen zu verstehen, also nicht nur die Herstellung von Schriftwerken, sondern auch die Fotografie, Filme sowie die bildende Kunst. ${ }^{85}$

(3) Wissenschaftliche Zwecke

Über Art. 9 Datenschutz-RL 95/46 hinausgehend nennt Art. 85 Abs. 2 DSGVO nun auch Datenverarbeitungen zu wissenschaftlichen Zwecken. Allerdings privilegiert Art. 85 Abs. 2 DSGVO wissenschaftliche Zwecke nicht umfassend, sondern bezieht sich allein auf den Bereich der Wissenschaftskommunikation. ${ }^{86}$

\section{bb) Umfang der Öffnungsklausel: Sanktionen?}

Nach Art. 85 Abs. 2 DSGVO haben die Mitgliedstaaten grundsätzlich Abweichungen und Ausnahmen von den gleichen Kapiteln vorzusehen, die auch schon nach Art. 9 Datenschutz-RL 95/46 ausgenommen waren, wenngleich zum Teil in anderer Anordnung und Nummerierung. ${ }^{87}$ Ebenso wie die Richtlinie verweist Art. 85 Abs. 2 DSGVO hingegen

81 Vgl. Specht/Bienemann, in: Sydow, Europäische Datenschutzgrundverordnung, DSGVO Art. 85 Rn. 13, 15: Journalistische Tätigkeit bei Privatpersonen nur bei „einer zweckgebundenen Funktionsäquivalenz zu journalistischen Tätigkeiten“ zu bejahen; Oster, in: Hartstein/Ring/u.a., RStV/ JMStV, Stand: August 2018; § 9c RstV Rn. 26, § 57 RStV Rn. 10.

82 Dazu s.u. V.3.

83 EuGH (GK), Urt. v. 13.5.2014, C-131/12, GRUR 2014, 895 Rn. 85 - Google Spain SL u. Google/ AEPD u. Costeja González.

84 Klein, Personenbilder im Spannungsfeld von Datenschutzgrundverordnung und Kunsturhebergesetz, 2017, S. 211.

85 Stender-Vorwachs, in: BeckOK Datenschutzrecht, 26. Edition, Stand: 1.8.2017, Art. 85 Rn. 16 ff.; enger Buchner/Tinnefeld, in: Kühling/Buchner, DS-GVO Art. 85 Rn. 23.

86 Golla, in: Specht/Mantz, Handbuch des Datenschutzrechts, 2018 (im Erscheinen), Teil C § 23 Rn. 6; Dix, in: Simitis/Hornung/Spiecker, Datenschutzrecht, 1. Aufl. 2019, Art. 85 Rn. 19.

87 Cornils, Das datenschutzrechtliche Medienprivileg unter Behördenaufsicht?, 2018, S. 32 f. 
nicht auf das Kapitel, das Rechtsbehelfe, Haftung und Sanktionen im Fall von Rechtsverstößen regelt, d.h. Kapitel VIII, und das über die Richtlinie hinausgehend nunmehr auch ein Beschwerderecht in Art. 77 DSGVO sowie Bußgeldsanktionen in Art. 83 DSGVO vorsieht.

Dies wirft zum einen die - den Umfang dieses Beitrags übersteigende - Frage auf, ob die in Kapitel VIII genannten Sanktionen, z.B. eine behördliche Aufsicht sowie Bußgeldsanktionen, auch bei Datenverarbeitungen zu journalistischen oder künstlerischen Zwecken grundsätzlich in Betracht kommen. ${ }^{88}$

Unklar ist darüber hinaus auch, ob im Anwendungsbereich des Art. 85 Abs. 2 DSGVO ein Anspruch auf Ersatz eines materiellen oder immateriellen Schadens aus Art. 82 DSGVO bestehen kann. Grundsätzlich gilt, dass der Schadensersatzanspruch nach Art. 82 DSGVO neben den Ansprüchen aus dem nationalen Recht besteht. ${ }^{89}$ Dies wirft die Frage auf, ob Art. 82 DSGVO die differenzierte deutsche Rechtsprechung, die die Sanktionen bei Verletzungen von Persönlichkeitsrechten zur Vermeidung von „chilling effects“ einschränkt, obsolet werden lässt, z.B. die Beschränkung des Geldentschädigungsanspruchs auf schwerwiegende Eingriffe in das Persönlichkeitsrecht, ${ }^{90}$ die Einschränkung der Gewährung von Geldentschädigungsansprüchen bei Persönlichkeitsverletzungen durch künstlerisches Schaffen ${ }^{91}$ sowie der Ausschluss von Ansprüchen auf materiellen Schadensersatz bei Persönlichkeitsrechtsverletzungen im Rahmen der redaktionellen Berichterstattung. ${ }^{92}$

Der Schadensersatzanspruch aus Art. 82 DSGVO knüpft an einen Verstoß „gegen diese Verordnung“ an. Fraglich ist daher, ob der Schadensersatzanspruch tatbestandlich überhaupt gegeben sein kann, wenn nicht unmittelbar gegen Vorschriften der Verordnung, sondern gegen im Rahmen einer Öffnungsklausel erlassene Vorschriften des nationalen Rechts verstoßen wird. Allerdings bestimmt der als Auslegungshilfe heranzuziehende Erw. 146 S. 5, dass ,zu einer Verarbeitung, die mit der vorliegenden Verordnung nicht im Einklang steht, (...) auch eine Verarbeitung [zählt], die nicht mit den nach Maßgabe der vorliegenden Verordnung erlassenen delegierten Rechtsakten und Durchführungsrechtsakten und Rechtsvorschriften der Mitgliedstaaten zur Präzisierung von Bestimmungen der vorliegenden Verordnung im Einklang steht“. Gegen die Einbeziehung von Verstößen gegen das auf Grundlage von Art. 85 Abs. 2 DSGVO weiterhin anwendbare nationale Äußerungsrecht spricht aber, dass dieses die DSGVO nicht präzisiert, sondern weiterhin einen eigenständigen Regelungskomplex im „mitgliedstaatlichen Reservatsbereich des inhaltlichen Medien- und

88 Dagegen mit überzeugenden Argumenten Cornils, Das datenschutzrechtliche Medienprivileg unter Behördenaufsicht?, 2018, S. $65 \mathrm{ff}$.

89 Quaas, in: Brink/Wolff, BeckOK Datenschutzrecht, 24. Edition, Stand: 1.5.2017, Art. 82 DSGVO Rn. 11.

90 BGH, Urt. v. 17.12.2013, VI ZR 211/12, ZUM-RD 2014, 145 Rn. 38 m. w. N.

91 BGH, Urt. v. 24.11.2009, VI ZR 219/08, GRUR 2010, 171 Rn. 13 f. - Esra mwN; LG Berlin, Urt. v. 3.6.2014, 27 O 56/14, ZUM 2014, 729, 732; Hildebrand, ZUM 2018, 585, 590.

92 BGH, Urt. v. 20.3.2012, VI ZR 123/11, ZUM 2012, 474 Rn. 28, 32 - Unfalltote; OLG Hamburg, Urt. v. 21.10.2008, 7 U 11/08, ZUM 2009, 65, 68. 
(allgemeinen) Äußerungsrechts“93 darstellt, in dem die Europäische Union keine umfassenden Kompetenzen besitzt. ${ }^{94}$ Das Äußerungsrecht präzisiert die DSGVO daher nicht, sondern schränkt sie i.S. der in Erw. 8 genannten zweiten Alternative ein. Daher erscheint es überzeugend, dass Art. 82 DSGVO bei Verletzungen des Rechts am eigenen Bild oder des allgemeinen Persönlichkeitsrechts nicht zur Anwendung kommt, sondern dass sich die zivilrechtlichen Sanktionen allein aus dem nationalen Recht ergeben. ${ }^{95}$ Eine endgültige Klärung dieser Frage kann aber nur durch den EuGH erfolgen.

Sollte der EuGH entgegen der hier vertretenen Ansicht von einer Anwendbarkeit des Art. 82 DSGVO ausgehen, so ergäbe sich m.E. aber auch aus den Unionsgrundrechten die Notwendigkeit, die Auswirkungen etwaiger Sanktionen auf die Ausübung der Kommunikationsfreiheiten und der Kunstfreiheit gemäß Art. 11 und Art. 13 GRCh sowie Art. 10 EMRK zu berücksichtigen. Dies hat auch die Ratspräsidentschaft in ihrem Kompromissvorschlag aus dem Jahr 2015 hinsichtlich der Formulierung des jetzigen Art. 85 DSGVO zum Ausdruck gebracht, in dem sie darauf hinwies, dass die Meinungsfreiheit als ein Element bei der Festlegung der Sanktion einbezogen werden und dass der Verhältnismäßigkeitsgrundsatz insoweit im Rahmen des Kapitels VIII reflektiert werden müsse. ${ }^{96}$ Auch der EuGH wäre demnach auf Grundlage der Kommunikationsfreiheiten und der Kunstfreiheit gemäß Art. 11 und Art. 13 GRCh sowie Art. 10 EMRK dazu gehalten zu gewährleisten, dass Sanktionen keine „,chilling effects“ entfalten.

\section{b) Datenverarbeitungen (auch) zu anderen Zwecken, Art. 85 Abs. 1 DSGVO}

Da die Öffnungsklausel des Art. 85 Abs. 2 DSGVO auf Datenverarbeitungen zu journalistischen, wissenschaftlichen, literarischen oder künstlerischen Zwecken beschränkt ist, stellt sich die Frage, welche rechtlichen Rahmenbedingungen für Veröffentlichungen zu anderen Zwecken gelten, z.B. bei Veröffentlichungen durch Vereine, bei Imagefilmen oder Werbe-

93 Cornils, Das datenschutzrechtliche Medienprivileg unter Behördenaufsicht?, 2018, S. 29.

94 Schiedermair, in: Ehmann/Selmayr, DS-GVO, 2. Auflage 2018, Art. 85 Rn. 9.

95 So bereits Bergt, in: Kühling/Buchner, DS-GVO Art. 82 Rn. 24; Gola/Piltz, in: Gola DatenschutzGrundverordnung, 2. Aufl. 2018, Art. 82 Rn. 16; Frenzel, in: Paal/Pauly, DS-GVO Art. 82 Rn. 9; a.A. noch Lauber-Rönsberg/Hartlaub, NJW 2017, 1057, 1060 f. Cornils weist zu Recht darauf hin, dass nationale Regelungen, die Schadensersatzansprüche bei einem Verstoß gegen von der Freistellung aufgenommene Regelungen der DSGVO begründen wollen, auch auf Art. 82 DSGVO als unmittelbar anwendbare Anspruchsgrundlage explizit hinweisen müssen, Cornils, Das datenschutzrechtliche Medienprivileg unter Behördenaufsicht?, 2018, S. 113.

96 Council of the European Union, Dok. v. 11.6.2015, Nr. 9788/15, S. 255 Rn. 627: „BE, DE, FR, IE and SE had requested to include also a reference to Chapter VIII. This was opposed to by COM. The Presidency points out that in case the freedom of expression prevails over the right to data protection, there will obviously no infringement to sanction. Where an infringement is found to have place, the interference with the freedom of expression will have to taken [sic!] into account as an element in the determination of the sanction. This application of the proportionality principle should be reflected in Chapter VIII.“ S. auch EuGH, Urt. v. 6.11.2003, C-101/01, EuZW 2004, 245, 247 Rn. 87 f. - Lindqvist. 
anzeigen von Unternehmen sowie bei rein privat motivierten Meinungsäußerungen in sozialen Netzwerken. Auch solche Kommunikationsprozesse werden nach der Rechtsprechung grundsätzlich durch die Kommunikationsfreiheiten geschützt. ${ }^{97}$

Art. 85 Abs. 1 DSGVO gibt den Mitgliedstaaten auf, das Recht auf den Schutz personenbezogener Daten mit dem Recht auf freie Meinungsäußerung und Informationsfreiheit durch Rechtsvorschriften in Einklang zu bringen. Die Regelung nimmt zwar ebenfalls auf die in Abs. 2 genannten Zwecke Bezug; diese sind aber nicht abschließend. ${ }^{98}$ Somit ist ihr Regelungsauftrag nicht auf Datenverarbeitungen zu journalistischen, wissenschaftlichen oder künstlerischen Zwecken beschränkt. Der Anwendungsbereich von Abs. 1 sowie derjenige von Abs. 2 überschneiden sich somit. ${ }^{99}$

In der Datenschutz-RL 95/46 hatte Art. 85 Abs. 1 DSGVO keine Entsprechung.

Es ist streitig, ob diese Regelung als gegenüber Abs. 2 eigenständige Öffnungsklausel interpretiert werden kann, auf deren Grundlage die Mitgliedstaaten weitere Ausnahmen von den datenschutzrechtlichen Vorgaben vorsehen können. Die Regelung des Art. 85 Abs. 1 DSGVO ist sowohl in ihrer deutschen als auch in der englischen und französischen Sprachfassung zweideutig. Es bleibt offen, ob ein Einklang hergestellt werden soll zwischen den beiden kollidierenden Grundrechten, das heißt dem Recht auf informationelle Selbstbestimmung einerseits und den Kommunikationsfreiheiten andererseits, oder ob sich dieser Regelungsauftrag lediglich darauf bezieht, dass die nationalen Regelungen des Rechts auf freie Meinungsäußerung und Informationsfreiheit mit den Regelungen der DSGVO in Einklang gebracht werden sollen.

aa) Art. 85 Abs. 1 DSGVO als bloßer Anpassungsauftrag?

Im Schriftum wird die Regelung zum Teil im letztgenannten Sinne als bloßer Auftrag zur Anpassung des nationalen Rechts an die DSGVO verstanden. ${ }^{100}$ Die DSGVO gebe inhaltlich das Niveau des Persönlichkeitsschutzes verbindlich vor. Art. 85 Abs. 1 DSGVO eröffne den Mitgliedstaaten - anders als Art. 85 Abs. 2 DSGVO - keine regulatorische Gestaltungsfreiheit und überlasse ihnen lediglich Ausführung und Mittelwahl. ${ }^{101}$ Gegen eine Interpreta-

97 BGH, Urt. v. 5.6.2008, I ZR 96/07, NJW 2008, 3782, 3783 - Zerknitterte Zigarettenschachtel; EGMR, Urt. v. 19.2.2015, 53649/09, NJW 2016, 781, 783 Rn. 45 - Ernst August von Hannover/ Deutschland zu Art. 10 EMRK.

98 Specht/Bienemann, in: Sydow, DSGVO, 2. Auflage 2018, Art. 85 Rn. 9; Cornils, Das datenschutzrechtliche Medienprivileg unter Behördenaufsicht?, 2018, S. 29 f.

99 Dix, in: Simitis/Hornung/Spiecker, Datenschutzrecht, 1. Aufl. 2019, Art. 85 Rn. 5.

100 Pötters, in: Gola, Datenschutz-Grundverordnung, 2. Auflage 2018, Art. 85 Rn. 5; Buchner/Tinnefeld, in: Kühling/Buchner DS-GVO, 2. Auflage 2018, Art. 85 Rn. 12; Kühling/Martini et al., Die DSGVO und das nationale Recht, $287 \mathrm{f}$; Pauly, in: Paal/Pauly, DS-GVO BDSG, 2. Auflage 2018, Art. 85 Rn. Rn. 4; Klein, Personenbilder im Spannungsfeld von Datenschutzgrundverordnung und Kunsturhebergesetz, 2017, S. 209.

101 Pauly, in: Paal/Pauly, DS-GVO BDSG, Art. 85 Rn. 4; Kühling/Martini et al., Die DSGVO und das nationale Recht, 286; Benecke/Wagner, DVB1 2016, 600, $602 \mathrm{f}$. 
tion als eigenständige Öffnungsklausel wird eingewandt, dass der Regelungsauftrag des Art. 85 Abs. 1 DSGVO zu wenig konkret sei und dass die in diesem Rahmen erlassenen Regelungen nicht der Meldepflicht gem. Abs. 3 unterliegen. Zudem ließe eine Interpretation des Art. 85 Abs. 1 DSGVO als spezialgesetzliche Öffnungsklausel das „fein auszisellierte Regelungssystem“ des Art. 6 Abs. 1-3 DSGVO obsolet werden. ${ }^{102}$ Daher enthalte Art. 85 Abs. 1 DSGVO lediglich einen Anpassungsauftrag, wonach die Mitgliedstaaten ihre bestehenden Vorschriften an die Vorgaben der DSGVO anzupassen hätten.

Die genannten Argumente sind jedoch nicht überzeugend. In Bezug auf Datenverarbeitungen durch Private enthält Art. 6 Abs. 1-3 DSGVO gerade kein ausdifferenziertes Regelungssystem. Vielmehr wäre die Zulässigkeit von Veröffentlichungen am Maßstab des Art. 6 Abs. 1 lit. f) DSGVO zu beurteilen, der eine Abwägung erfordert, jedoch keine Abwägungskriterien vorgibt. ${ }^{103}$ Dagegen hat zumindest die deutsche Rechtsprechung auf Grundlage von $\S 23$ KUG bzw. dem allgemeinen Persönlichkeitsrecht ein umfangreiches „Case law" entwickelt.

Gewichtiger ist das Argument, dass die Mitteilungspflicht nach Art. 85 Abs. 3 DSGVO nur mitgliedstaatliche Regelungen nach Abs. 2 erfasst. Die Bedeutung dieses Arguments relativiert sich jedoch, wenn man die Entstehungsgeschichte der Norm berücksichtigt. Die Mitteilungspflicht war stark umstritten und wurde erst auf den letzten Verhandlungsmetern in die Endfassung aufgenommen. ${ }^{104}$ Abs. 3 entspricht im Wesentlichen der Formulierung, die er bereits im Kommissionsentwurf ${ }^{105}$ hatte, der aber nur den jetzigen Abs. 2 beinhaltete. Insofern lässt sich Abs. 3 auch so interpretieren, dass es angesichts der Eile des Gesetzgebungsverfahrens versäumt wurde, die Mitteilungspflicht auf auf Abs. 1 gestützte Regelungen zu erstrecken. ${ }^{106}$

Auch das Argument, den Mitgliedstaaten werde im Rahmen des Art. 85 Abs. 1 DSGVO lediglich die Ausführung und Mittelwahl überlassen, ist wenig überzeugend, da die Methodik für die Beurteilung der Zulässigkeit der Datenverarbeitung - nämlich eine konkrete Abwägung der betroffenen Interessen im Einzelfall - durch die Rechtsprechung des EuGH ${ }^{107}$

102 Kühling/Martini et al., Die DSGVO und das nationale Recht, $287 \mathrm{f}$.

103 S. zu weiterer Kritik an Art. 6 DSGVO Roßnagel/Nebel/Richter, ZD 2015, 455, 457: „dieser reichlich unscharfe Tatbestand"; Schulz, in: Gola, Datenschutz-Grundverordnung, 2. Auflage 2018, Art. 6 Rn. 60: „fehlende Rechtssicherheit der Norm“.

104 Abs. 3 wurde erst in die finale Kompromissfassung vom 15.12.2015, Dok. Nr. 15039/15 (S. 200) eingefügt und fehlte noch im Vorschlag der Ratspräsidentschaft vom 27.11.2015, Dok. Nr. 14481/15.

105 S. Art. 80 DSGVO-E des Vorschlags für eine Verordnung des Europäischen Parlaments und des Rates zum Schutz natürlicher Personen bei der Verarbeitung personenbezogener Daten und zum freien Datenverkehr (Datenschutz-Grundverordnung), KOM/2012/011 endg.

106 S. dazu ausführlich Cornils, Das datenschutzrechtliche Medienprivileg unter Behördenaufsicht?, 2018, S. $53 \mathrm{f}$.

107 EuGH, Urt. v. 19.10.2016, C-582/14, ZUM 2016, 1024 Rn. 62 - Breyer/Deutschland. 
und auch des EGMR ${ }^{108}$ vorgegeben ist. Im Gegensatz dazu kann das Schutzniveau bei einer Einzelfallabwägung jedoch kaum abstrakt im Vorhinein festgelegt werden.

Gegen die Interpretation als Anpassungsauftrag spricht zudem, dass die DSGVO in ihrem Anwendungsbereich das nationale Recht ohnehin verdrängt, soweit keine Öffnungsklausel einschlägig ist, sodass sich die Sinnhaftigkeit eines Anpassungsauftrags nicht erschließt. ${ }^{109}$ Zudem enthält die DSGVO - mit Ausnahme des Art. 17 Abs. 3 DSGVO - auch keine speziellen Vorgaben für Kommunikationsvorgänge, an die die Mitgliedstaaten ihre bestehenden Vorschriften anpassen könnten.

\section{bb) Einschränkung des mitgliedstaatlichen Gestaltungsspielraums im Rahmen anderer Öffnungsklauseln?}

Denkbar wäre allenfalls, Art. 85 Abs. 1 DSGVO so auszulegen, dass er einen Auftrag an die Mitgliedstaaten enthält, im Rahmen der ihnen zustehenden Gestaltungsspielräume bei der Ausgestaltung der sonstigen Öffnungsklauseln der Verordnung eine Konkordanz zwischen dem Schutz personenbezogener Daten und den Kommunikationsfreiheiten herzustellen. So wäre z.B. denkbar, dass diese Erwägungen eine Beschränkung der Informationsrechte der Betroffenen gemäß Art. 13 DSGVO auf Grundlage der Öffnungsklausel des Art. 23 Abs. 1 lit. i) DSGVO legitimieren. So kann es z.B. bei Fotografien von großen Menschenmengen oder von Bauwerken oder sonstigen Sehenswürdigkeiten, auf denen dem Fotografen unbekannte Menschen als „Beiwerk“ zu sehen sind, für diesen schwierig sein, die Informationsrechte nach Art. 13 zu erfüllen.

Wenn man den Anwendungsbereich des Art. 85 Abs. 1 DSGVO auf die Ausgestaltung der anderen Öffnungsklauseln reduzieren würde, wäre dies zwar mit dem Wortlaut der Regelung vereinbar. Der Umstand, dass ihr dann lediglich ein sehr geringer Anwendungsbereich verbliebe, spricht angesichts der Entstehungsgeschichte der Regelung gegen eine solche Auslegung. Der Vorschlag der Kommission beinhaltete zunächst nur eine an Art. 9 Datenschutz-RL 95/46 angelehnte Regelung. ${ }^{110}$ Sowohl das Europäische Parlament als auch der Rat strebten jedoch eine Erweiterung der Privilegierung an, um eine Vereinbarkeit zwischen dem Recht auf Schutz personenbezogener Daten und den Kommunikationsfreiheiten auch in den Fällen zu erreichen, in denen andere als journalistische, wissenschaftliche oder künstlerische Zwecke verfolgt werden. Die von ihnen unterbreiteten Vorschläge beschränkten sich daher auf eine allgemeine, mit Art. 85 Abs. 1 DSGVO vergleichbare Öffnungsklau-

108 S. z.B. EGMR, Urt. v. 7.2.2012 (GK), Nr. 40660/08, NJW 2012, 1053, Ziff. 108 ff. - von Hannover/Deutschland II; EGMR, Urt. v. 19.10.2017, 71233/13, NJW 2018, 3083 Rn. 34 - Fuchsmann/ Deutschland.

109 Cornils, Das datenschutzrechtliche Medienprivileg unter Behördenaufsicht?, 2018, S. 47 f.; Ziebarth/Elsaß, ZUM 2018, 578, 582.

110 S. Art. 80 DSGVO-E des Vorschlags für eine Verordnung des Europäischen Parlaments und des Rates zum Schutz natürlicher Personen bei der Verarbeitung personenbezogener Daten und zum freien Datenverkehr (Datenschutz-Grundverordnung), KOM/2012/011 endg. 
sel. ${ }^{111}$ Die endgültige Fassung vereint also als Kompromiss sowohl den Kommissionsvorschlag als auch die allgemeine Abwägungsklausel des Europäischen Parlaments und des Rates. Dies spricht dafür, dass beiden Absätzen des Art. 85 DSGVO eine eigenständige normative Bedeutung zukommt. ${ }^{112}$

\section{cc) Art. 85 Abs. 1 DSGVO als eigenständige Öffnungsklausel}

Auch aufgrund seiner Entstehungsgeschichte ist es am überzeugendsten, Art. 85 Abs. 1 DSGVO als eigenständige, weitere Öffnungsklausel zu interpretieren, die den Mitgliedstaaten eine zusätzliche, über die in Art. 85 Abs. 2 DSGVO genannten Zwecke hinausgehende Regelungsbefugnis eröffnet. ${ }^{113}$

Zum anderen ist hier nochmals darauf hinzuweisen, dass auch eine kommunikative Betätigung zu nicht-journalistischen Zwecken, z.B. Veröffentlichungen durch Vereine, privat motivierte Meinungsäußerungen in sozialen Netzwerken und Veröffentlichungen zu Werbezwecken, grundsätzlich durch die Kommunikationsfreiheiten geschützt werden. Wenn die Öffnungsklausel des Art. 85 auf die in Abs. 2 genannten Zwecke beschränkt wäre, würden hiervon nicht erfasste Meinungsäußerungen den Vorgaben der DSGVO unterfallen. Insbesondere bei Privaten würden z.B. die umfangreichen Informationspflichten des Art. 13 DSGVO sowie die Grundsätze des Art. 5 DSGVO zu einer erheblichen Einschränkung der Kommunikationsfreiheiten führen. Auch vor diesem Hintergrund erscheint es überzeugend, Art. 85 Abs. 1 DSGVO als Öffnungsklausel zu interpretieren, um „eventuelle Lücken im Schutzkonzept der enumerativ formulierten Öffnungsklausel des Art. 85 Abs. 2 DSGVO“ zu schließen. ${ }^{114}$ Dies entspricht auch der Absicht des Europäischen Parlaments, das mit der weiten Fassung der Freistellung gerade klarstellen wollte, dass ,alle Aspekte der Meinungsfreiheit erfasst werden, nicht nur die der Journalisten, Künstler oder Schriftsteller“. ${ }^{115}$

111 S. Art. 80 DSGVO-E der Legislativen Entschließung des Europäischen Parlaments vom 12.3.2014, P7_TA(2014)0212; Art. 80 DSGVO-E in der Allgemeinen Ausrichtung des Rates vom 11.6.2015, Dok. Nr. 9565/15 umfasste sowohl die allgemeine Abwägungsklausel des Abs. 1 als auch die konkrete Handlungsverpflichtung des Abs. 2, hingegen noch nicht die Mitteilungspflicht des Abs. 3. S. dazu auch Cornils, Das datenschutzrechtliche Medienprivileg unter Behördenaufsicht?, 2018, S. $37 \mathrm{ff}$.

112 So überzeugend Cornils, Das datenschutzrechtliche Medienprivileg unter Behördenaufsicht?, 2018, S. 43.

113 Ebenso Schulz/Heilmann, in: Gierschmann/Schlender/Stentzel/Veil, DSGVO, 2017, Art. 85 Rn. 7; Cornils, Das datenschutzrechtliche Medienprivileg unter Behördenaufsicht?, 2018, S. 45 ff.; Hoidn, in: Roßnagel, Europäische Datenschutz-Grundverordnung, 2017, § 4 Rn. 180; s. auch Lauber-Rönsberg/Hartlaub, NJW 2017, 1057, 1061 f.; dies., in: Götting/Schertz/Seitz, Handbuch des Persönlichkeitsrechts, 2. Aufl. 2018 (im Erscheinen), § 22 Rn. 41 ff.

114 Cornils, Das datenschutzrechtliche Medienprivileg unter Behördenaufsicht?, 2018, S. 63.

115 Committee on Civil Liberties, Justice and Home Affairs, Draft Report on the proposal for a regulation of the European Parliament and of the Council on the protection of individual with regard to the processing of personal data and on the free movement of such data (General Data Protection Regulation) (COM (2012)0011 - C7-0025/2012 - 2012/0011 (COD)); 16.1.2013, 
Darüber hinaus würde eine Begrenzung der Freistellung auf journalistische Nutzungen $\mathrm{zu}$ Abgrenzungsproblemen führen, da sich z.B. bei einer Vereinspublikation nicht immer zweifelsfrei feststellen lassen wird, ob sie noch als journalistischen Zwecken dienend einzuordnen ist.

Ein Verständnis des Art. 85 Abs. 1 DSGVO als eigenständige Öffnungsklausel zieht die Frage nach sich, in welchem Verhältnis diese Regelung zu Art. 85 Abs. 2 DSGVO steht. Es erscheint überzeugend, den tatbestandlich weiteren Abs. 1 als allgemeine Öffnungsklausel $\mathrm{zu}$ interpretieren, der den Mitgliedstaaten einen größeren Freiraum einräumt, während Abs. 2 als Mindestgarantie aufzufassen ist, der besonders schutzbedürftige Verarbeitungssituationen erfasst. ${ }^{116}$

\section{Auswirkungen auf das Äußerungsrecht}

Fraglich ist nun, welche Auswirkungen die Neuordnung des Datenschutzrechts auf das Äußerungsrecht hat. Hierbei geht es einerseits um die Verzahnung von Unionsrecht und nationalem Recht, andererseits um das Zusammenspiel von Bundes- und Landesrecht an der Schnittstelle zwischen Bürgerlichem Recht und Öffentlichem Recht.

\section{Datenverarbeitungen zu journalistischen Zwecken}

Im Rahmen der Reformen zur Anpassung des BDSG an die DSGVO hat der Bundesgesetzgeber aus kompetenzrechtlichen Gründen keine $\S 41$ Abs. 1 BDSG a.F. entsprechende Regelung erlassen, da seit der Förderalismusreform und dem Entfallen der Rahmengesetzgebungskompetenz für das Pressewesen nunmehr ausschließlich die Länder zuständig sind. Die Begründung zum RegE ging aber davon aus, dass auch weiterhin eine Freistellung von Datenverarbeitungen zu journalistischen Zwecken durch die Landesgesetzgeber erfolgen wird. ${ }^{117}$

\section{a) Regelungen im Landespresserecht}

In der Tat haben alle Bundesländer Regelungen zur Freistellung von Datenverarbeitungen zu journalistischen und literarischen Zwecken in ihre Presse- bzw. Mediengesetze aufge-

Änderungsantrag 324, S. 193. A.A. Specht/Bienemann, in: Sydow, Europäische Datenschutzgrundverordnung, 2. Auflage 2018, Rn. 9, die sich dagegen wenden, Art. 85 Abs. 1 DSGVO als ,allgemeines Meinungsprivileg“ zu verstehen.

116 S. dazu näher Cornils, Das datenschutzrechtliche Medienprivileg unter Behördenaufsicht?, 2018, S. 60 .

117 Entwurf eines Gesetzes zur Anpassung des Datenschutzrechts an die Verordnung (EU) 2016/679 und zur Umsetzung der Richtlinie (EU) 2016/680 (Datenschutz-Anpassungs- und -Umsetzungsgesetz EU - DSAnpUG-EU, BR-Drs. 110/17, S. 73 f. 
nommen. ${ }^{118}$ Die Freistellungen in den Pressegesetzen erfassen z.T. weiterhin nur Unternehmen der Presse und deren Hilfsunternehmen, ${ }^{119}$ obgleich die Ausgestaltungsverpflichtung des Art. 85 Abs. 2 DSGVO aufgrund des weiten Verständnisses der journalistischen Tätigkeit ${ }^{120}$ nicht auf diesen Personenkreis beschränkt ist. Auch diese Länder werden dem Ausgestaltungsauftrag aber gerecht, wenn sie an anderer Stelle, z.B. in den Landesdatenschutzgesetzen, zusätzlich eine allgemeine Freistellung von Datenverarbeitungen für journalistische Zwecke vorsehen. ${ }^{121}$

Hinsichtlich des Umfangs der Freistellung ist festzustellen, dass alle Länder die entsprechenden Datenverarbeitungen von den Kapiteln II-VII und IX mit Ausnahme der Art. 5 Abs. 1 lit. f), 24, 32 DSGVO freistellen ${ }^{122}$ und eine Verpflichtung zum Datengeheimnis statuieren, was der früheren Rechtslage entspricht. Damit schöpfen die Länder die Freistellungsmöglichkeiten nicht voll aus. Eine Verpflichtung zur Beibehaltung der Art. 5 Abs. 1 lit. f), 24, 32 DSGVO zur Gewährleistung der Datensicherheit besteht nach der DSGVO nicht, da die Mitgliedstaaten nach Art. 85 Abs. 2 DSGVO auch Ausnahmen von diesen Regelungen vorsehen können. ${ }^{123}$ Zum Teil sehen die Regelungen zudem spezielle Betroffenenrechte vor, insbes. Auskunftsansprüche. ${ }^{124}$

Interessant ist die Frage, inwieweit Rechtsverstöße durch Schadensersatzansprüche sanktioniert werden können. Die meisten Länder stellen Datenverarbeitungen zu journalistischen Zwecken zwar nicht generell von den in Kapitel VIII vorgesehenen Sanktionen frei, ${ }^{125}$ beschränken jedoch die Haftung auf Schadensersatz aus Art. 82 DSGVO auf Verstö-

118 Für den Pressebereich s. § 12 LPresseG B-W; 11 Bayrisches PresseG und Art. 38 BayDSG; § 19 BlnDSG; § 16a BbgLPresseG und § 29 BbgDSG; § 5 Bremer Pressegesetz; § 11a Hamburgisches PresseG i.V.m. §37 Medienstaatsvertrag HSH; § 10 Hessisches PresseG; § 18a LPresseG M-V und § 12 DSG-V; § 19 Niedersächsisches PresseG; § 12 LPresseG NRW; § 12 LMedienG R-P; $\S 11$ Saarländisches MedienG; § 11a Sächsisches PresseG; § 10a LPresseG LSA; § 10 LPresseG $\mathrm{S}-\mathrm{H}$; § 11a Thüringer PresseG.

119 Z.B. § 12 Abs. 2 LPresseG B-W; Art. 11 Bayrisches PresseG; § 16a BbgLPresseG; § 19 Niedersächsisches PresseG; § 11a Thüringer PresseG; allgemein journalistische Zwecke privilegieren $\S 12$ LPresseG NRW; § 12 LMedienG R-P; § 11 Saarländisches MedienG; § 11a Sächsisches PresseG; $§ 10$ LPresseG S-H.

120 Dazu s.o. IV.3.a) aa).

121 S. z.B. Art. 38 Abs. 1 BayDSG; § 29 BbgDSG; § 25 ThürDSG.

$122 \S 11$ a Sächsisches PresseG ordnet lediglich die Anwendbarkeit von Art. 5 Abs. 1 lit. f) DSGVO an.

123 Cornils, Das datenschutzrechtliche Medienprivileg unter Behördenaufsicht?, 2018, S. 108.

124 Allerdings bestehen Zweifel an der Vereinbarkeit des Auskunftsanspruchs z.B. gemäß $\$ 57$ Abs. 2 S. 1 und 2 RStV mit der Pressefreiheit, s. Oster, in: Hartstein/Ring/u.a., RStV/JMStV, Stand: August 2018, $§ 57$ RstV Rn. $20 \mathrm{ff}$.

125 Zum Teil soll auch das gesamte Kapitel VIII nicht anwendbar sein, wenn Presseunternehmen dem Pressekodex und der Beschwerdeordnung des Deutschen Presserates unterliegen, s. z.B. $\S 37$ Medienstaatsvertrag HSH. Kritisch unter dem Gesichtspunkt der Benachteiligung von Bürgerjournalisten und Bloggern Kahl/Piltz, K\&R 2018, 289, 293. Allerdings ist m.E. fraglich, ob eine behördliche Aufsicht über Datenverarbeitungen zu journalistischen Zwecken mit der Presse- 
ße gegen die wenigen nach der DSGVO fortbestehenden Verpflichtungen aus Art. 5 Abs. 1 lit. f), 24, 32 DSGVO sowie auf die Pflicht zur Wahrung des Datengeheimnisses. Dies ist m.E. mit den Vorgaben der Verordnung vereinbar, obgleich Art. 85 Abs. 2 DSGVO seinem Wortlaut nach keine Abweichungen bzw. Ausnahmen von Kapitel VIII zulässt. Jedoch kann eine Haftung auf Schadensersatz aus Art. 82 DSGVO nur bei Verstößen gegen die DSGVO sowie gem. Erw. 146 S. 5 gegen die Verordnung präzisierende mitgliedstaatliche Regelungen gegeben sein. Wie oben dargelegt, lösen dagegen Verstöße gegen das nationale Äußerungsrecht nur die im nationalen Recht vorgesehenen Sanktionen aus, dagegen keine Schadensersatzansprüche aus Art. 82 DSGVO, da das Äußerungsrecht keine Präzisierung der DSGVO darstellt. ${ }^{126}$

Da die Vorgaben der DSGVO aber mit Ausnahme der genannten Regelungen vollständig derogiert werden, wird auch der Anwendungsbereich des Schadensersatzanspruchs entsprechend eingeschränkt. Die Hinweise auf Art. 82 DSGVO bringen dies - letztlich nur deklaratorisch - zum Ausdruck. Aus Gründen der Rechtssicherheit ist dieser Hinweis aber sinnvoll. ${ }^{127}$

\section{b) Regelungen im Äußerungsrecht}

Neben den mediendatenschutzrechtlichen Regelungen auf Landesebene ist wie dargelegt zudem das bundesrechtliche Äußerungsrecht als „,materielles Datenschutzrecht“ einschlägig und gewährleistet in diesem Sinne einen Datenschutz außerhalb der Datenschutzgesetze. ${ }^{128}$ Die von einer Medienberichterstattung Betroffenen bleiben somit nicht ohne rechtlichen Schutz. ${ }^{129}$

Zugleich ist nicht von der Hand zu weisen, dass viele Regelungsbereiche der DSGVO in den fragmentarischen äußerungsrechtlichen Regelungen keine Entsprechung finden. So statuiert $\S 22 \mathrm{KUG}$ zwar ein grds. Einwilligungserfordernis. § $23 \mathrm{KUG}$ regelt gesetzliche Erlaubnistatbestände, die funktional durchaus mit Art. 6 Abs. 1 lit. a) und f) DSGVO vergleichbar sind. Weitere Elemente, z.B. die grundsätzliche Unwiderruflichkeit der Einwilli-

freiheit vereinbar ist, s. Cornils, Das datenschutzrechtliche Medienprivileg unter Behördenaufsicht?, 2018, S. $65 \mathrm{ff}$.

126 S. o. IV. 3.a) bb).

127 Anders Cornils, Das datenschutzrechtliche Medienprivileg unter Behördenaufsicht?, 2018, S. 113, der die Erwähnung des Art. 82 DSGVO für erforderlich hält, wenn beabsichtigt ist, Verstöße gegen die DSGVO durch einen Schadensersatzanspruch zu sanktionieren.

128 Vgl. dazu bereits Garstka JZ 1978, 507, 511.

129 A.A. aber Buchner/Tinnefeld, in: Kühling/Buchner, DS-GVO BDSG, 2. Auflage 2018, DSGVO, Art. 85 Rn. 31 (,pauschale Freistellung von den materiell-rechtlichen Datenverarbeitungsvorgaben“), Rn. 32 („,Regelungsdefizit“); Stender-Vorwachs, in: Brink/Wolff/, BeckOK Datenschutzrecht, 26. Edition, Stand: 1.8.2017, Art. 85 Rn. 33. 
gung, ${ }^{130}$ die Doppelzuständigkeit bei Einwilligungen „einsichtsfähiger“ Minderjähriger ${ }^{131}$ und das Erfordernis der Informiertheit der Einwilligung ${ }^{132}$ wurden durch Gerichtsentscheidungen geprägt, sind jedoch rudimentär geblieben. Andere Themenkomplexe, wie der Bereich der technischen und organisatorischen Maßnahmen (z.B. Art. 25 DSGVO) oder spezielle Anforderungen bei Übermittlungen an Drittstaaten (Art. 44 ff. DSGVO), finden kein Äquivalent.

Fraglich ist daher, ob die äußerungsrechtlichen Regelungen inhaltlich den Vorgaben der Art. 85 Abs. 2 DSGVO genügen. M.E. ist dies zu bejahen. ${ }^{133}$ Die Regelung ermöglicht Ausnahmen und Abweichungen von den zentralen Kapiteln der DSGVO, ohne insoweit materiell-rechtliche Vorgaben zu machen, soweit dies zur Herstellung einer praktischen Konkordanz erforderlich ist. Dass den Mitgliedstaaten durch Art. 85 Abs. 2 DSGVO ein weiter Spielraum zugestanden wird ${ }^{134}$, kommt auch dadurch zum Ausdruck, dass der europäische Gesetzgeber in diesem Bereich keine Vollharmonisierung anstrebt, wie sich aus Erw. 153 DSGVO ergibt.

\section{aa) Recht am eigenen Bild}

Die Anwendbarkeit des KUG für Bildnisveröffentlichungen zu journalistischen Zwecken wurde nun in ersten Entscheidungen vom OLG Köln bestätigt. ${ }^{135}$ Streitig ist in Bezug auf das KUG als Altregelung aber, ob insoweit auch eine Notifizierungspflicht nach Art. 85 Abs. 3 DSGVO besteht. ${ }^{136}$

Das OLG Köln bejaht die Vereinbarkeit des KUG mit den Vorgaben des Art. 85 Abs. 2 DSGVO. Zutreffend führt das Gericht aus, dass die DSGVO im Kern keine materiell-recht-

130 BAG, Urt. v. 11.12.2014, 8 AZR 1010/13, NJW 2015, 2140 Rn. 37 ff. - Einwilligung des Arbeitnehmers.

131 S. statt vieler Specht, in: Dreier/Schulze, UrhG, 6. Aufl. 2018, § 22 KUG Rn. 26.

132 S. statt vieler Specht, in: Dreier/Schulze, UrhG, 6. Aufl. 2018, § 22 KUG Rn. 19.

133 A.A. Buchner/Tinnefeld, in: Kühling/Buchner, DS-GVO BDSG, 2. Auflage 2018, DSGVO, Art. 85 Rn. 31 f.; Stender-Vorwachs, in: Brink/Wolff/, BeckOK Datenschutzrecht, 26. Edition, Stand: 1.8.2017, Art. 85 Rn. 33; Benedikt/Kranig, ZD 2019, 4, 5f.; s. auch die Entschließung der DSK „Umsetzung der DSGVO im Medienrecht“ vom 9.11.2017.

134 Pötters, in: Gola, Datenschutz-Grundverordnung, 2. Auflage 2018, Art. 85 Rn. 16.

135 OLG Köln, Beschl. v. 18.6.2018, 15 W 27/18, ZUM-RD 2018, 549 Rn. 6 m. Anm. Lauber-Rönsberg; OLG Köln, Beschl. v. 8.10.2018, 15 U 110/10, BeckRS 2018, 26059 Rn. 12; dagegen Benedikt/Klein, ZD 2019, 4, 5 f.; zweifelnd hinsichtlich der Fortgeltung des KUG als „Altregelung“ auch Klein, Personenbilder im Spannungsfeld von Datenschutzgrundverordnung und Kunsturhebergesetz, 2017, S. 181 ff. mangels entsprechender Willensäußerung des Gesetzgebers.

136 Verneinend Schulz/Heilmann, in: Gierschmann/Stender/Stentzel/Veil, DSGVO, 2017, Art. 85 Rn. 66; Schlender-Vorwachs, in: Brink/Wolff, BeckOK Datenschutzrecht, 24. Edition, Stand: 1.8.2017, Art. 85 Rn. 32; bejahend Grages, in: Plath, DSGVO/BDSG, 3. Aufl. 2018, Art. 85 Rn. 11; Pauly, in: Paal/Pauly, DS-GVO/BDSG, 2. Aufl. 2018, Art. 85 Rn. 14; Dix, in: Simitis/ Hornung/Spiecker, Datenschutzrecht, 1. Aufl. 2019, Art. 85 Rn. 30; Benedikt/Kranig, ZD 2019, 4,5 . 
lichen Vorgaben macht und in diesem Bereich keine Vollharmonisierung anstrebt, wie auch Erw. 153 DSGVO zum Ausdruck bringt, sondern nur die Herstellung einer praktischen Konkordanz zwischen dem Datenschutz einerseits und den Kommunikationsfreiheiten andererseits fordere. ${ }^{137}$ Insofern gesteht Art. 85 Abs. 2 DSGVO den Mitgliedstaaten einen weiten Spielraum zu. ${ }^{138}$ Vor diesem Hintergrund ist es überzeugend, dass das KUG trotz seiner im Vergleich zur DSGVO geringen Regelungsdichte angesichts seiner Auslegungsoffenheit, insbesondere aufgrund der unbestimmten Rechtsbegriffe wie dem Begriff des „Bildnisses aus dem Bereiche der Zeitgeschichte“ gemäß $§ 23$ Abs. 1 und Abs. 2 KUG, diesen Anforderungen gerecht wird. ${ }^{139}$

Überzeugend geht das OLG Köln davon aus, dass die Abwägung nicht mehr allein nach Maßgabe der deutschen Grundrechte, sondern nunmehr auch unter Berücksichtigung der unionsrechtlichen Grundrechtspositionen erfolgen müsse. ${ }^{140}$ Da sich die deutsche Rechtsprechung auch bislang schon an den Vorgaben der EMRK orientiert hat, die nach Art. 52 Abs. 3 GRCh auch für die DSGVO als Rechtserkenntnisquelle heranzuziehen ist, ${ }^{141}$ wird dies voraussichtlich nicht zu großen Abweichungen von der bisherigen Rechtsprechung führen. Zwar hatte die italienische Ratspräsidentschaft im Laufe des Gesetzgebungsverfahrens die Einführung eines Erwägungsgrundes vorgeschlagen, wonach dem Datenschutzrecht grds. Vorrang vor dem Informationsinteresse von Internetnutzern zukommen solle. ${ }^{142}$ Dieser Vorschlag konnte sich jedoch nicht durchsetzen.

Aufgrund der umfassenden Freistellungen der Datenverarbeitungen im journalistischen Kontext von den Vorgaben der DSGVO durch die Medienprivilegien in den Landesmedienbzw. Landesdatenschutzgesetzen können auch die übrigen Regelungen der DSGVO, z.B. zu Transparenz und den Betroffenenrechten, nicht ergänzend herangezogen werden, um das fragmentarische KUG zu ergänzen. ${ }^{143}$ Angesichts der bestehenden Rechtsunsicherheit wäre aber eine Klarstellung durch den Gesetzgeber sehr wünschenswert.

137 OLG Köln, Beschl. v. 18.6.2018, 15 W 27/18, ZUM-RD 2018, 549 Rn. 8 f.

138 Pötters, in: Gola, Datenschutz-Grundverordnung, 2. Auflage 2018, Art. 85 Rn. 16.

139 So auch Ziebarth/Elsaß, ZUM 2018, 578, 584.

140 OLG Köln, Beschl. v. 18.6.2018, 15 W 27/18, ZUM-RD 2018, 549 Rn. 9; s. dazu auch Schiedermair, in: Ehmann/Selmayr, DSGVO, 2017, Art. 85 Rn. 8; Oster, in: Hartstein/Ring/u.a., RStV/ JMStV, Stand: August 2018, § 9c RstV Rn. 8 f.; a.A. Cornils, Das datenschutzrechtliche Medienprivileg unter Behördenaufsicht?, 2018, S. 85 ff.

141 Dazu Kühling/Martini et al., Die DSGVO und das nationale Recht, $288 \mathrm{f}$.

142 Presidency, Proposal General Data Protection Regulation - Right to be forgotten and the Google judgment, 11289/1/14 REV 1, http://register.consilium.europa.eu/doc/srv?l=EN\&f=ST\%2011289 \%202014\%20REV\%201; s. auch EuGH GRUR 2014, 895 Rn. 81 - Google Spain.

143 A.A. wohl Benedikt/Kranig, ZD 2019, 4, 6. 


\section{bb) Allgemeines Persönlichkeitsrecht}

Fraglich ist, ob auch der zivilrechtliche Schutz des allgemeinen Persönlichkeitsrechts weiterhin unverändert anwendbar ist. Das allgemeine Persönlichkeitsrecht gemäß § 823 Abs. 1 BGB i.V.m. Art. 2 Abs. 1, Art. 1 Abs. 1 GG bietet Schutz gegenüber der Verwendung von Persönlichkeitsmerkmalen außerhalb des Bildnisbereichs sowie gegenüber der Aufnahme von Bildern, die ebenfalls nicht von $\S \S 22$ ff. KUG erfasst wird. ${ }^{144}$

Das OLG Köln führt in seiner Entscheidung als obiter dictum aus, dass auch das allgemeine Persönlichkeitsrecht im Rahmen der Öffnungsklausel des Art. 85 Abs. 2 DSGVO weiterhin Anwendung finde. ${ }^{145}$ Dieser Auffassung ist voll und ganz zuzustimmen. Die gegenteiligen Stellungnahmen der Landesdatenschutzbeauftragten ${ }^{146}$ verkennen, dass zwischen dem verfassungsrechtlichen allgemeinen Persönlichkeitsrecht und seiner zivilrechtlichen Gewährleistung zu differenzieren ist. ${ }^{147}$ Dies wird schon durch den Umstand belegt, dass die Anerkennung des zivilrechtlichen allgemeinen Persönlichkeitsrechts durch den BGH in der grundlegenden Leserbrief-Entscheidung bereits im Jahr $1954^{148}$ und damit vor der Anerkennung des verfassungsrechtlichen allgemeinen Persönlichkeitsrechts durch das BVerfG erfolgte. ${ }^{149}$ Auch wenn die deutschen Grundrechte im Anwendungsbereich der DSGVO durch die Unionsgrundrechte, insbesondere Art. 7 und Art. 8 GRCh, überlagert werden, wie die genannten Stellungnahmen der Datenschutzbeauftragten anführen, ändert dies nichts an dem Fortbestand des zivilrechtlichen allgemeinen Persönlichkeitsrechts. Allenfalls könnte die Geltung der Unionsgrundrechte zur Folge haben, dass das durch $\S 823$ Abs. 1 BGB geschützte zivilrechtliche allgemeine Persönlichkeitsrecht seine verfassungsrechtliche Verankerung in diesem Kontext nunmehr nicht allein in Art. 2 Abs. 1 GG i. V. m. Art. 1 Abs. 1 GG, sondern auch in Art. 7 und Art. 8 GRCh findet. Anders als die deutschen Grundrechte wird das zivilrechtliche allgemeine Persönlichkeitsrecht m.E. jedoch - ebenso wie das spezielle KUG - nicht durch die DSGVO verdrängt, soweit es sich als Regelung zum Ausgleich von Persönlichkeitsschutz und Kommunikationsfreiheiten in die Öffnungsklauseln des Art. 85 DSGVO einpasst. Inhaltlich stellt es ebenso wie das Recht am eigenen

144 Götting, in: Schricker/Loewenheim, Urheberrecht, 5. Aufl. 2017, § 22 Rn. 34.

145 OLG Köln, Beschl. v. 18.6.2018, 15 W 27/18, ZUM-RD 2018, 549 Rn. 9 unter Hinweis auf Schulz/Heilmann, in: Gierschmann/Schlender/Stentzel/Veil, DSGVO, 2017, Art. 85 Rn. 8.

146 Brandenburg, Verarbeitung personenbezogener Daten bei Fotografien - Rechtliche Anforderungen unter der DS-GVO, https://www.lda.brandenburg.de/media_fast/4055/RechtlicheAnforderun genFotografie.pdf (zuletzt abgerufen am 5.11.2018), S. 4; Hamburg, Rechtliche Bewertung von Fotografien einer unüberschaubaren Anzahl von Menschen nach der DSGVO außerhalb des Journalismus, https:/www.filmverband-suedwest.de/wp-content/uploads/2018/05/Vermerk_DSGVO. pdf (zuletzt abgerufen am 5.11.2018), S. 3 f.; ebenso Ziebarth/Elsaß, ZUM 2018, 578, 584; Benedikt/Kranig ZD 2019, 4, 5.

147 S. näher Götting, in: ders./Schertz/Seitz, Handbuch des Persönlichkeitsrechts, 2. Aufl. 2019 , § 3.

148 BGH, Urt. v. 25.5.1954, I ZR 211/53, NJW 1954, 1404 - Leserbrief.

149 Dazu Baston-Vogt, Der sachliche Schutzbereich des zivilrechtlichen allgemeinen Persönlichkeitsrechts, 1997, S. 119 f. 
Bild ein geeignetes Regelungsinstrument dar, um eine praktische Konkordanz zwischen dem Schutz personenbezogener Daten einerseits und den Kommunikationsfreiheiten andererseits herzustellen. Auch hier wäre eine Klärung durch den deutschen Gesetzgeber höchst wünschenswert, die letztlich eine Kodifikation des richterrechtlich geprägten allgemeinen Persönlichkeitsrechts bedeuten würde. Zwar würde eine Kodifikation aufgrund der stets erforderlichen Abwägung im Einzelfall hinsichtlich der Zulässigkeit der Verwendung von Persönlichkeitsmerkmalen insoweit keinen wesentlichen Gewinn an Rechtssicherheit bedeuten. In Bezug auf die von der Rechtsprechung geprägten Abwägungsparameter sowie weitere Fragen - z.B. hinsichtlich des Verhältnisses zur DSGVO, aber auch hinsichtlich anderer Einzelfragen wie der Widerruflichkeit von Einwilligungserklärungen - könnte eine Kodifikation aber durchaus zu mehr Transparenz und damit auch zu mehr Rechtssicherheit beitragen.

\section{Datenverarbeitungen zu künstlerischen Zwecken}

Noch sehr viel weniger klar ist die Rechtslage hinsichtlich Datenverarbeitungen zu künstlerischen Zwecken, z.B. in Bezug auf Fotografen, Filmemacher oder Schriftsteller, die reale Personen abbilden bzw. als Vorlage für ihr künstlerisches Schaffen wählen.

\section{a) Regelungen in den Landesdatenschutzgesetzen}

Soweit ersichtlich haben acht Bundesländer Parallelregelungen zu den Medienprivilegien eingeführt und auch Datenverarbeitungen für künstlerische Zwecke von den datenschutzrechtlichen Vorgaben weitgehend - mit Ausnahme der Art. 5 Abs. 1 lit. f), 24, 32 DSGVO freigestellt. Auch diese Regelungen beschränken die Schadensersatzpflicht aus Art. 82 DSGVO auf Verstöße gegen die wenigen verbleibenden datenschutzrechtlichen Pflichten hinsichtlich technischer bzw. organisatorischer Maßnahmen. ${ }^{150}$

Dies wirft zum einen die Frage auf, ob diese „Kunstprivilegien“ nur Datenverarbeitungen durch öffentliche Stellen erfassen oder ob sie auch Datenverarbeitungen nicht-öffentlicher Stellen einbeziehen. ${ }^{151}$ Im ersten Fall käme den Regelungen ein äußerst geringer Anwendungsbereich zu. Wenn die „Kunstprivilegien“ auch nicht-öffentliche Stellen privilegieren, ist es andererseits zweifelhaft, ob eine Gesetzgebungskompetenz der Länder zum Erlass entsprechender Regelungen besteht. ${ }^{152}$ Grundsätzlich ist der Datenschutz im nicht-öffentlichen Bereich dem Recht der Wirtschaft gemäß Art. 74 Abs. 1 Nr. 11 GG zuzuordnen,

$150 \S 19$ LDSG G B-W; Art. 38 BayDSG; § 19 BlnDSG; § 29 BbgDSG; § 12 Hamburgisches DSG; $\S 12$ DSG M-V; § 19 DSG NRW; § 11a Thüringer DSG.

151 So ordnen Art. 1 Abs. 1 S. 4 BayDSG, § 2 Abs. 7 BlnDSG, § 29 Abs. 3 BbgDSG, § 12 Abs. 3 Hamburgisches DSG, § 2 Abs. 1 S. 3 Thüringer DFG an, dass die Freistellungen der Datenverarbeitungen auch für nicht öffentliche Stellen gelten.

152 S. auch Kahl/Piltz, K\&R 2018, 289, 294. 
aus dem sich eine konkurrierende Gesetzgebungskompetenz ergibt. ${ }^{153}$ Zwar steht die Gesetzgebungskompetenz im Bereich der Kunst grundsätzlich aufgrund ihrer Kulturhoheit den Bundesländern zu. ${ }^{154}$ Es ist jedoch sehr fraglich, ob die hier relevante Materie des „Kunstdatenschutzrechts" tatsächlich schwerpunktmäßig dem Kulturrecht zuzuordnen ist. Überzeugender ist es, das Verhältnis zwischen dem Künstler und der betroffenen Person, z.B. die Zulässigkeit der künstlerischen Verarbeitung von Persönlichkeitsmerkmalen ohne Einwilligung, als eine Frage der Ausgestaltung des Persönlichkeitsschutzes zwischen Privaten anzusehen und sie daher dem Gebiet des bürgerlichen Rechts gemäß Art. 74 Abs. 1 Nr. 1 GG zuzuordnen, sodass sie der konkurrierenden Gesetzgebung unterfällt. ${ }^{155}$ Hierfür spricht auch, dass die Gesetzgebungszuständigkeit des Bundes für die den Konflikt bereits adressierende Regelung des $\S 23$ Abs. 1 Nr. 4 KUG in der Vergangenheit nicht in Frage gestellt wurde.

Eine Einordnung in die konkurrierende Gesetzgebung hätte zur Folge, dass die Länderkompetenz erlischt, Art. 72 Abs. 1 GG, wenn der Bund von seinem Zugriffsrecht Gebrauch gemacht und eine Materie abschließend geregelt hat. Vieles spricht dafür, dass die Länder daher z.B. keine Gesetzgebungskompetenzen haben, um Erlaubnistatbestände für bildliche Darstellungen zu regeln, da diese schon durch $§ 23$ KUG abschließend geregelt werden. Insofern ist die Wirksamkeit der in den „Kunstprivilegien“ vorgesehenen Derogation von Art. 6 DSGVO zweifelhaft; m.E. könnte diese Einschränkung des Geltungsanspruchs der DSGVO nur durch den Bundesgesetzgeber erfolgen.

\section{b) Regelungen im Äußerungsrecht}

Unabhängig hiervon stellt sich die Frage, ob die äußerungsrechtlichen Regelungen des KUG und des allgemeinen Persönlichkeitsrechts als „Abweichungen oder Ausnahmen“ i.S. des Art. 85 Abs. 2 DSGVO fortgelten, auch wenn sie keine explizite Freistellung von den Vorgaben der DSGVO enthalten. Aus den oben angeführten Gründen ist auch im künstlerischen Kontext davon auszugehen, dass das Äußerungsrecht aufgrund seiner Struktur inhaltlich dazu geeignet ist, den Rahmen des Art. 85 Abs. 2 DSGVO auszufüllen.

Allerdings stellt sich aufgrund der spärlichen Regelungsdichte und mangels expliziter Bezugnahme auf die DSGVO auch in diesem Zusammenhang die Frage, ob die Regelungen des Äußerungsrechts abschließend sind, ob sie also auch die Anwendung von Regelungen

153 Kühling/Klar/Sackmann, Datenschutzrecht, 4. Aufl. 2018, Rn. 56.

154 Uhle, in: Maunz/Dürig, GG, 83. EL April 2018, Art. 70 Rn. 106.

155 Die Gesetzgebungskompetenz für das KUG wird z.T. auch aus der ausschließlichen Gesetzgebungskompetenz für Gewerblichen Rechtsschutz, Urheber- und Verlagsrecht gemäß Art. 73 Abs. 1 Nr. 9 abgeleitet, s. Uhle, in: Maunz/Dürig, GG, 83. EL April 2018, Art. 73 Rn. 202. Da das KUG seit Aufhebung der urheberrechtlichen Regelungen zum 1.1.1966 keine urheberrechtlichen Regelungen i.e.S. enthält, ist dies indes wenig überzeugend. 
der DSGVO „sperren“, zu denen sie keine funktionale Entsprechung enthalten. ${ }^{156}$ Ein Beispiel hierfür sind die Betroffenenrechte der Art. $12 \mathrm{ff}$. DSGVO. Hier könnte das Schweigen des KUG (und erst recht des nicht kodifizierten allgemeinen Persönlichkeitsrechts!) einerseits als „beredtes Schweigen“ i.S. einer Freistellung von den Vorgaben der DSGVO, andererseits aber auch so interpretiert werden, dass in diesem Bereich die Regelungen der DSGVO gelten sollen. So wird im Schrifttum z.B. ein Rückgriff auf die DSGVO in Erwägung gezogen, da das KUG keine speziellen Regelungen zu Aufsichtsbehörden, Verantwortlichen und Auftragsverarbeitern enthalte. ${ }^{157}$

Zwar spricht der Umstand, dass das Äußerungsrecht nicht nur lange vor der DSGVO, sondern auch lange vor den ersten nationalen Datenschutzgesetzen in Kraft getreten ist, dafür, dass es eine abschließende Regelung der Materie bezweckt. Um Rechtssicherheit zu schaffen - sowohl hinsichtlich des abschließenden Charakters des Äußerungsrechts als auch im Verhältnis zur DSGVO -, wäre eine explizite Klarstellung durch den Gesetzgeber jedoch wünschenswert. Dies gilt nicht nur für das Recht am eigenen Bild, sondern auch für das allgemeine Persönlichkeitsrecht.

\section{Datenverarbeitungen zu nicht in Art. 85 Abs. 2 DSGVO aufgeführten Zwecken}

Große Unklarheit besteht über das anwendbare Rechtsregime, wenn ein Kommunikationsvorgang nicht den durch Art. 85 Abs. 2 DSGVO privilegierten journalistischen, wissenschaftlichen oder künstlerischen Zwecken dient, sondern wenn es sich z.B. um Maßnahmen der Öffentlichkeitsarbeit oder eine rein privat motivierte Meinungsäußerung in sozialen Netzwerken handelt. Wie oben dargestellt ist streitig, ${ }^{158}$ ob Art. 85 Abs. 1 DSGVO für solche Kommunikationsvorgänge eine eigenständige Öffnungsklausel vorsieht und ob das Äußerungsrecht insoweit weiterhin Anwendung finden kann. ${ }^{159}$

\section{a) Szenario 1: Art.85 Abs. 1 DSGVO als Öffnungsklausel}

Wenn man mit der hier vertretenen Ansicht Art. 85 Abs. 1 DSGVO als Öffnungsklausel ansieht, dann wirft dies - analog zu den im künstlerischen Kontext diskutierten Erwägungen die Frage auf, ob die äußerungsrechtlichen Regelungen des KUG und des allgemeinen Per-

156 Bei der soeben diskutierten Fallgruppe der Datenverarbeitung für journalistische Zwecke ergibt sich diese Problematik nicht, da diese aufgrund der wirksamen Medienprivilegien im Landesrecht von den datenschutzrechtlichen Vorgaben freigestellt wird. Dagegen haben nicht alle Bundesländer Freistellungen für den künstlerischen Bereich eingeführt. Zudem ist die Wirksamkeit der „Kunstprivilegien“ fraglich, so dass unsicher ist, ob Datenverarbeitungen zu künstlerischen Zwecken von den datenschutzrechtlichen Vorgaben tatsächlich wirksam freigestellt sind.

157 Klein, Personenbilder im Spannungsfeld von Datenschutzgrundverordnung und Kunsturhebergesetz, 2017, S. 231.

158 S.o. IV.3.b).

159 Offen gelassen von LG Frankfurt, Urt. v. 13.9.2018, 2-03 O 283/18, BeckRS 2018, 23105, Rn. $20 \mathrm{ff}$. 
sönlichkeitsrechts genügen, um, wie von Art. 85 Abs. 1 DSGVO gefordert, als „Rechtsvorschriften“ das Recht auf den Schutz personenbezogener Daten mit der Meinungsäußerungsund Informationsfreiheit in Einklang zu bringen. Auch hier gilt, dass das Äußerungsrecht sich bislang inhaltlich durchaus als geeigneter Ausgleichsmechanismus erwiesen hat.

Jedoch besteht hier ebenfalls die Problematik, dass den bereits lange vor Inkrafttreten der DSGVO bestehenden Regelungen in ihrer jetzigen Form nicht zweifelsfrei entnommen werden kann, inwieweit sie eine Einschränkung des Geltungsanspruchs der DSGVO beabsichtigen oder ob Teilbereiche der DSGVO, z.B. die Betroffenenrechte, weiterhin zur Anwendung kommen. Auch aus diesem Grund wäre, analog zu den für künstlerische Darbietungen geltenden Überlegungen, eine explizite Klarstellung durch den Gesetzgeber wünschenswert.

\section{b) Szenario 2: Art. 85 Abs. 1 DSGVO keine Öffnungsklausel}

Wenn der EuGH Art. 85 Abs. 1 DSGVO jedoch - aus seiner Sicht zuständigkeitserweiternd - so interpretieren würde, dass diese Regelung keine Öffnungsklausel darstellt, dann wären die nicht von Art. 85 Abs. 2 DSGVO erfassten Sachverhalte nach den Vorgaben der DSGVO zu beurteilen, soweit der sachliche Anwendungsbereich der Verordnung eröffnet ist. Dagegen ergeben sich die rechtlichen Rahmenbedingungen für journalistische Datenverarbeitungen, wie oben dargestellt, allein aus dem nationalen Äußerungsrecht, so dass hier unterschiedliche Vorgaben gelten. Auch außerhalb des Anwendungsbereichs der DSGVO gilt das KUG wie dargestellt weiterhin, z.B. für Abbildungen Verstorbener sowie für Datenverarbeitungen zu persönlichen und familiären Tätigkeiten. ${ }^{160}$

\section{aa) Zulässigkeit von Datenverarbeitungen}

Die Wirksamkeit einer Einwilligung würde sich in diesem Fall nach Art. 6 Abs. 1 lit. a), Art. 7 DSGVO richten. ${ }^{161}$ Als Erlaubnistatbestände, die eine Datenverarbeitung ohne Einwilligung des Betroffenen gestatten, kämen zudem Art. 6 Abs. 1 lit. b) und f) DSGVO in Betracht. Es ist zu vermuten, dass der EuGH ähnliche Kriterien wie die in $\S 23 \mathrm{KUG}$ geregelten Fallgruppen in die Abwägung einbeziehen wird. ${ }^{162}$ Problematisch wäre allerdings unter dem Gesichtspunkt der Rechtssicherheit, dass es einige Jahre oder gar Jahrzehnte dauern dürfte, bis sich eine gefestigte Rechtsprechung des EuGHs zu speziellen Fallgestal-

$160 \mathrm{Zu}$ dem bei analogen Fotos eingeschränkten Anwendungsbereich der DSGVO s. Klein, Personenbilder im Spannungsfeld von Datenschutzgrundverordnung und Kunsturhebergesetz, 2017 , S. $29 \mathrm{ff}$.

161 Dagegen sind Personenbilder nach überzeugender Ansicht nicht pauschal als Daten i.S. von Art. 9 DSGVO einzuordnen, Klein, Personenbilder im Spannungsfeld von Datenschutzgrundverordnung und Kunsturhebergesetz, 2017, S. $26 \mathrm{ff}$.

162 LG Frankfurt, Urt. v. 13.9.2018, 2-03 O 283/18, BeckRS 2018, 23105, Rn. 35; Specht, in: Dreier/ Schulze, UrhG, 6. Aufl. 2018, §23 KUG Rn. 1. 
tungen, z.B. der Verwendung von Persönlichkeitsmerkmalen in der satirischen Werbung ohne Einwilligung des Betroffenen, entwickelt haben wird. Da Art. 6 Abs. 1 lit. f) DSGVO keine Öffnungsklausel ist, ${ }^{163}$ würden dem deutschen Gesetzgeber keine Gestaltungsspielräume zur Verfügung stehen, um die Vorgaben zu präzisieren.

Zudem müssten Datenverarbeiter die Betroffenenrechte der Art. 12 ff. DSGVO beachten. Insbesondere die Informationspflicht nach Art. 13 DSGVO kann in der Praxis durchaus zu gravierenden Problemen führen, wenn eine Person eine große Menschenmenge fotografiert oder wenn bei einem Foto einer Sehenswürdigkeit dem Fotografen unbekannte Menschen als „Beiwerk“ zu sehen sind. Wie bereits erwähnt, besteht grundsätzlich auch in diesen Fällen eine Informationspflicht nach Art. 13 DSGVO, sofern hier nicht Art. 14 DSGVO einschlägig ist oder die Informationspflicht nach Art. 11 Abs. 1 DSGVO entfällt. Anders als Art. 14 DSGVO in seinem Abs. 5 lit. b sieht Art. 13 DSGVO keine Einschränkung unter Verhältnismäßigkeitsgesichtspunkten vor. Eine den Vorgaben des Art. 13 DSGVO entsprechende Information dürfte dem Fotografen aber in der Vielzahl solcher Fälle unmöglich sein. Hier besteht also die Gefahr, dass die umfassend ausgestalteten Betroffenenrechte die Ausübung der Meinungs- und Informationsfreiheit gefährden. Grundsätzlich ist die Entscheidung des europäischen Gesetzgebers, die Betroffenenrechte zu stärken, anzuerkennen. Wenn aber Datenverarbeitungen zu dem Zweck einer Meinungsäußerung erfolgen und damit selbst grundrechtlichen Schutz durch die Meinungs- und Informationsfreiheit genießen, muss auch hier eine praktische Konkordanz der betroffenen Rechtsgüter hergestellt werden.

Bisherige Stellungnahmen der Landesdatenschutzbeauftragten versuchen, diese Problematik durch eine extensive Auslegung des Anwendungsbereichs des Art. 14 DSGVO zu „entschärfen“. ${ }^{164}$ Darüber hinaus wäre es m.E. wünschenswert, wenn der Gesetzgeber den ihm nach Art. 23 Abs. 1 lit. i) DSGVO zustehenden Gestaltungsspielraum ${ }^{165}$ nutzen würde, um diese Vorgaben kommunikationsgerecht auszugestalten für den Fall, dass der EuGH Art. 85 Abs. 1 DSGVO nicht als eigenständige Öffnungsklausel ansieht. Dies gilt insbesondere für die Informationspflichten und Auskunftsansprüche. Hinsichtlich der Löschungsansprüche der betroffenen Person hat der europäische Gesetzgeber selbst für eine entspre-

163 Buchner/Petri, in: Kühling/Buchner, DS-GVO BDSG, 2. Auflage 2018, Art. 6 Rn. 143.

164 So der Vermerk des Hamburgischen Beauftragten für Datenschutz und Informationssicherheit, „Rechtliche Bewertung von Fotografien einer unüberschaubaren Anzahl von Menschen nach der DSGVO außerhalb des Journalismus“ abrufbar unter https://www.filmverband-suedwest.de/wpcontent/uploads/2018/05/Vermerk_DSGVO.pdf, zuletzt abgerufen am 5.11.2018, sowie die Stellungnahme der Landesbeauftragten für den Datenschutz des Landes Brandenburg, Verarbeitung personenbezogener Daten bei Fotografien - Rechtliche Anforderungen unter der DS-GVO, abrufbar unter https://www.lda.brandenburg.de/media_fast/4055/RechtlicheAnforderungenFotografie.pdf, zuletzt abgerufen am 5.11.2018.

165 Art. 23 Abs. 1 lit. i) gestattet den Mitgliedstaaten eine Beschränkung u.a. zum Schutz der Rechte und Freiheiten anderer Personen. Als „,andere Personen“ kommt neben Dritten nach überzeugender, aber umstrittener Ansicht auch der Verantwortliche, d.h. der Datenverarbeiter selbst, in Betracht, s. Bäcker, in: Kühling/Buchner, DS-GVO, 2. Auflage 2018, Art. 23 Rn. 32; s. dazu auch Lauber-Rönsberg, in: Specht/Mantz, Handbuch Datenschutzrecht, 2018 (im Erscheinen), §5 Rn. $128 \mathrm{f}$. 
chende Einschränkung in Art. 17 Abs. 3 lit. a) DSGVO gesorgt. Dafür, dass den nationalen Gesetzgebern entsprechende Gestaltungsspielräume zustehen, spricht neben der Öffnungsklausel des Art. 23 Abs. 1 lit. i) DSGVO als weiteres Argument in diesem Zusammenhang auch Art. 85 Abs. 1 DSGVO.

\section{bb) Folgefragen}

Wenn Art. 85 Abs. 1 DSGVO nicht als eigenständige Öffnungsklausel zu interpretieren wäre, so könnten $\S \S 22$ ff. KUG und der zivilrechtliche Schutz des Persönlichkeitsrechts wie dargestellt nur im Rahmen des durch Art. 85 Abs. 2 DSGVO ermöglichten Regelungsspielraums für journalistische, wissenschaftliche und künstlerische Zwecke sowie außerhalb des Anwendungsbereichs der DSGVO für Abbildungen Verstorbener sowie für Datenverarbeitungen zu persönlichen und familiären Tätigkeiten aufrechterhalten werden.

Datenverarbeitungen für andere Zwecke wären hingegen nicht nach dem Äußerungsrecht, sondern allein nach den datenschutzrechtlichen Vorgaben zu beurteilen. Soweit sich also die Anwendungsbereiche der DSGVO und des KUG überschneiden, würde das KUG durch die DSGVO verdrängt. Grundsätzlich gilt, dass Regelungen des nationalen Rechts, denen aufgrund des Anwendungsvorrangs einer Verordnung kein eigenständiger Anwendungsbereich mehr verbleibt, zur Vermeidung von Rechtsunsicherheiten aufzuheben sind. ${ }^{166}$ Dies könnte dafür sprechen, den Anwendungsbereich der äußerungsrechtlichen Regelungen auf die in Art. 85 Abs. 2 DSGVO genannten Zwecke sowie den Schutz Verstorbener sowie auf Datenverarbeitungen zu persönlichen und familiären Tätigkeiten einzuschränken. Zugleich zeigt sich, dass dieses Szenario zu erheblichen Abgrenzungsschwierigkeiten führen würde.

Fraglich ist darüber hinaus, ob tatsächlich eine vollständige Kongruenz zwischen dem Äußerungsrecht und der DSGVO hinsichtlich der Schutzgüter besteht. Das Datenschutzrecht dient wohl dem Schutz der Privatsphäre und der Gewährleistung von informationeller Selbstbestimmung des Einzelnen. ${ }^{167}$ Dies trifft auch auf den Persönlichkeitsschutz zu. Allerdings umfasst dieser zusätzlich noch weitere Schutzrichtungen, insbesondere den Schutz der Ehre ${ }^{168}$ als Domäne der nationalen Gesetzgeber. Diese Überlegungen könnten dafür sprechen, dass selbst für den Fall, dass das nationale Äußerungsrecht außerhalb des journalistischen, künstlerischen und wissenschaftlichen Bereichs weitgehend durch die DSGVO überlagert werden sollte, auch weiterhin ein Bedarf für einen nationalen Persönlichkeitsschutz neben der DSGVO bestehen könnte. Allerdings würde der nationale Persönlichkeitsschutz nur in solchen Fällen relevant, in denen ein Eingriff in Persönlichkeitsrechte zwar

166 S. allgemein zu nationalen Regelungen, denen kein eigenständiger Anwendungsbereich verbleibt Kühling/Martini et al., Die DSGVO und das nationale Recht, 2016, 3.

167 S. allerdings Veil, NVwZ 2018, 686, 690 ff. zu der Frage des Schutzguts als eines der „Mysterien des Datenschutzrechts“.

168 S. z.B. BVerfG NJW 2018, 1744 Rn. 19 - Straßenfotografie; Specht, in: Dreier/Schulze, UrhG, 6. Aufl. 2018, § 23 KUG Rn. 26. 
datenschutzrechtlich zulässig, jedoch wegen eines Verstoßes gegen den Schutz der Ehre unzulässig ist. Auch hier dürfte eine Abgrenzung im Einzelfall jedoch sehr schwierig sein, so dass eine solche restriktive Auslegung des Art. 85 Abs. 1 DSGVO zahlreiche weitere Fragen aufwerfen würde..

\section{Fazit}

Art. 85 Abs. 2 DSGVO enthält eine Öffnungsklausel für Datenverarbeitungen zu journalistischen Zwecken sowie zu wissenschaftlichen, künstlerischen oder literarischen Zwecken.

Mit dem OLG Köln ist davon auszugehen, dass auch nach Wirksamwerden der DSGVO sowohl das KUG als auch das zivilrechtliche allgemeine Persönlichkeitsrecht gemäß $\S 823$ Abs. 1 BGB i.V. m. Art. 2 Abs. 1, Art. 1 Abs. 1 GG für journalistische Tätigkeiten weiterhin anwendbar sind. Zwar sind im Rahmen der Abwägung nunmehr neben den nationalen Grundrechten auch die Unionsgrundrechte zu berücksichtigen. Da dies aber bislang schon der Fall ist, wird dieser Umstand voraussichtlich nicht zu einer wesentlichen Änderung der Abwägungsparameter führen. Damit ändert sich für Datenverarbeitungen zu journalistischen Zwecken nach der hier vertretenen Ansicht wenig.

Allerdings werden im Unterschied zur früheren Rechtslage nunmehr von fast allen Bundesländern - im Einklang mit Art. 85 Abs. 2 DSGVO - nicht nur Presseunternehmen und ihre Hilfsunternehmen von den datenschutzrechtlichen Vorgaben freigestellt, sondern allgemein die Datenverarbeitungen zu journalistischen Zwecken privilegiert, sodass der Kreis der Begünstigten erweitert wurde. Erfasst werden daher auch redaktionell nicht eingebundene, selbstständige Journalisten. Offen ist weiterhin, ob auch andere Akteure wie z.B. Blogger, Plattformbetreiber etc. unter die Freistellungen fallen.

Rechtsunsicherheiten bestehen auch im Hinblick auf Datenverarbeitungen für künstlerische Zwecke. Zwar spricht vieles dafür, dass sich über das Erfordernis zur Berücksichtigung der Unionsgrundrechte hinaus keine wesentlichen Änderungen im Vergleich zur früheren Rechtslage ergeben. Allerdings spricht vieles dafür, dass die von den Bundesländern eingeführten Regelungen zur Freistellung von Datenverarbeitungen von den datenschutzrechtlichen Vorgaben nichtig sind, da insoweit der Bundesgesetzgeber durch das Äußerungsrecht auf Grundlage von Art. 74 Abs. 1 Nr. 1 GG eine abschließende Regelung getroffen hat. Um mehr Rechtssicherheit zu schaffen, wäre aber eine explizite Regelung durch den deutschen Gesetzgeber sinnvoll, die - sowohl im Verhältnis zu den Landesregelungen als auch zur DSGVO - den abschließenden Charakter des Äußerungsrechts im Spannungsfeld zwischen dem Persönlichkeitsschutz und der Kunstfreiheit klarstellt. Es wäre aus Gründen der Transparenz und Rechtssicherheit wünschenswert, wenn dies zugleich zu einer Kodifikation des im künstlerischen Kontext ebenfalls relevanten allgemeinen Persönlichkeitsrechts genutzt würde.

Auch im Hinblick auf Datenverarbeitungen im Zusammenhang mit sonstigen Meinungsäußerungen, die zwar nicht als journalistisch eingeordnet, aber dennoch von der Meinungsfreiheit geschützt werden, z.B. im Rahmen der Öffentlichkeitsarbeit oder bei privat 
motivierten Kommunikationsvorgängen, bestehen gravierende Rechtsunsicherheiten. Diese Datenverarbeitungen können nur auf Art. 85 Abs. 1 DSGVO gestützt werden. Unklar ist allerdings, ob es sich bei dieser Regelung um eine eigenständige Öffnungsklausel handelt. Wird dies mit der hier vertretenen Ansicht bejaht, so würde ebenfalls eine weitgehende Kontinuität mit der früheren Rechtslage gewahrt. Auch hier wäre aber eine explizite Regelung sinnvoll, um Rechtssicherheit zu schaffen. Sieht man hingegen Art. 85 Abs. 1 DSGVO nicht als Öffnungsklausel an, so wären diese Datenverarbeitungen nach den Vorgaben der DSGVO zu beurteilen. In diesem Fall sollte das KUG durch eine Reform explizit auf den ihm dann verbleibenden Anwendungsbereich der Verwendung von Bildnissen zu journalistischen, wissenschaftlichen, künstlerischen oder literarischen Zwecken beschränkt werden. 